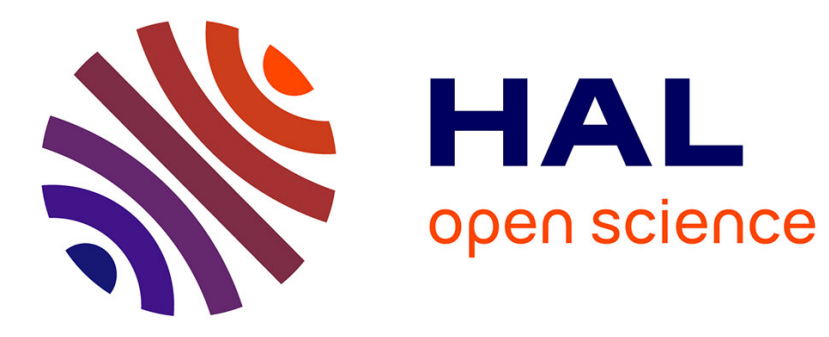

\title{
Optimal absorption of acoustical waves by a boundary
}

Frédéric Magoulès, Thi Phuong Kieu Nguyen, Pascal Omnes, Anna

Rozanova-Pierrat

\section{To cite this version:}

Frédéric Magoulès, Thi Phuong Kieu Nguyen, Pascal Omnes, Anna Rozanova-Pierrat. Optimal absorption of acoustical waves by a boundary. SIAM Journal on Control and Optimization, 2021, 59 (1), pp.561-583. 10.1137/20M1327239 . hal-01558043v6

\section{HAL Id: hal-01558043 \\ https://hal.science/hal-01558043v6}

Submitted on 17 Jul 2020

HAL is a multi-disciplinary open access archive for the deposit and dissemination of scientific research documents, whether they are published or not. The documents may come from teaching and research institutions in France or abroad, or from public or private research centers.
L'archive ouverte pluridisciplinaire HAL, est destinée au dépôt et à la diffusion de documents scientifiques de niveau recherche, publiés ou non, émanant des établissements d'enseignement et de recherche français ou étrangers, des laboratoires publics ou privés. 


\title{
Optimal absorption of acoustic waves by a boundary
}

\author{
Frédéric Magoulès* $^{*} \quad$ Thi Phuong Kieu Nguyen ${ }^{\dagger} \quad$ Pascal Omnes $^{\ddagger}$ \\ Anna Rozanova-Pierrat ${ }^{\S}$
}

\begin{abstract}
In the aim to find the simplest and most efficient shape of a noise absorbing wall to dissipate the acoustical energy of a sound wave, we consider a frequency model described by the Helmholtz equation with a damping on the boundary. The well-posedness of the model is shown in a class of domains with $d$-set boundaries $(N-1 \leq d<N)$. We introduce a class of admissible Lipschitz boundaries, in which an optimal shape of the wall exists in the following sense: We prove the existence of a Radon measure on this shape, greater than or equal to the usual Lebesgue measure, for which the corresponding solution of the Helmholtz problem realizes the infimum of the acoustic energy defined with the Lebesgue measure on the boundary. If this Radon measure coincides with the Lebesgue measure, the corresponding solution realizes the minimum of the energy. For a fixed porous material, considered as an acoustic absorbent, we derive the damping parameters of its boundary from the corresponding time-dependent problem described by the damped wave equation (damping in volume).
\end{abstract}

Keywords: Absorbing wall; wave propagation; shape optimization; Helmholtz equation; sound absorption; Robin boundary condition.

\section{Introduction}

The diffraction and absorption of waves by a system with both absorbing properties and irregular geometry is an open physical problem. This has to be solved to understand why anechoic chambers (electromagnetic or acoustic) do work better with irregular absorbing walls. The first studies relating irregular geometry and absorption are performed numerically in [24]. Therefore there is a question about the existence of an optimal shape of an absorbent wall (for a fixed absorbing material), optimal in the sense that it is as dissipative as possible for a large range of frequencies, and at the same time that such a wall could effectively be constructed. In the framework of the propagation of acoustic waves, the acoustic absorbent material of the wall is considered as a porous medium. In this article, for a fixed frequency of the sound wave, we solve the shape optimization problem that consists in minimizing the acoustic energy for a frequency model with a damping on the boundary. Then we extend this method in order to find an efficient shape for a finite range of frequencies.

In the area of optimization of acoustic performances of non absorbing walls, Duhamel [19, $20]$ studies sound propagation in a two-dimensional vertical cut of a road wall and uses genetic algorithms to obtain optimal shapes (some of them are however not connected and thus could not be easily manufactured). The author also uses a branch and bound (combinatorial optimization) type linear programming in order to optimize the sensors' positions

\footnotetext{
* MICS, CentraleSupélec, Université Paris Saclay, 3 rue Joliot Curie, 91190 Gif-sur-Yvette, France and Faculty of Engineering and Information Technology, University of Pécs, Pécs, Hungary.

$\dagger$ MICS, CentraleSupélec, Université Paris-Saclay, France.

¥DES-Service de thermo-hydraulique et de mécanique des fluides (STMF), CEA, Université Paris-Saclay, F-91191, Gif-sur-Yvette, France and Université Sorbonne Paris Nord, LAGA, CNRS, UMR 7539, F-93430, Villetaneuse, France.

§CentraleSupélec, Université Paris-Saclay, France (correspondence, anna.rozanovapierrat@centralesupelec.fr).
} 
that allow an active noise control, following former work introduced by Lueg [26] in 1934. Abe et al. [1] consider a boundary elements based shape optimization of a non absorbing two-dimensional wall in the framework of a two-dimensional sound scattering problem for a fixed frequency (for the Helmholtz equation) using a topological derivative with the principle that a new shape or topology is obtained by nucleating small scattering bodies. Also for the Helmholtz equation for a fixed frequency, using the shape derivative of a functional representing the acoustical energy, Cao and Stanescu [11] consider a two-dimensional shape design problem for a non-absorbing part of the boundary to reduce the amount of noise radiated from aircraft turbofan engines. For the same problem, Farhadinia [23] developed a method based on measure theory, which does not require any information about gradients and the differentiability of the cost function.

On the other hand, for shape optimization problems there are theoretical results, reviewed in Refs. [4, 37], which rely on the topological derivatives of the cost functional to be minimized, with numerical application of the gradient method in both two and three dimensional cases (in the framework of solid mechanics). In particular, Achdou and Pironneau [2] considered the problem of optimization of a photocell, using a complex-valued Helmholtz problem with periodic boundary conditions with the aim to maximize the solar energy in a dissipative region. For acoustic waves in the two-dimensional case, optimization of the shape of an absorbing inclusion placed in a lossless acoustic medium was considered in Refs. [38, 39]. The considered model is the linear damped wave equation [15, 8]. Using the topology derivative approach, Münch et al. consider in [38, 39] the minimization of the acoustic energy of the solution of the damped wave equation at a given time $T>0$ without any geometric restrictions and without the purpose of the design of an absorbent wall. See also [6] for the shape optimization of shell structure acoustics.

In this article, we study the two-dimensional shape optimization problem for a Helmholtz equation with a damping on the boundary, modeled by a complex-valued Robin boundary condition. The shape of the damping boundary is to be found, in the aim to minimize the total acoustical energy of the system. In Section 2, we introduce the frequency model and its time-dependent analogue with a dissipation on the boundary. We analyze its dissipative properties and give the well-posedness results, due to [9, 25] for at least Lipschitz boundaries, but we generalize the results for the Helmholtz problem in a larger class of domains with $d$-set boundaries using [7, 40] (see Appendix A). This class, named in [7] "admissible domains" and containing for instance the Von Koch fractals, is composed of all Sobolev extension domains, thanks to results of [27], with boundaries on which it is possible to define a surjective linear continuous trace operator with linear continuous right inverse. However, for the shape optimization problem, only the Lipschitz boundary case is considered here.

We compare the frequency model with dissipation by the boundary to the corresponding model with a dissipation in the volume. Dissipation in the volume is described by a damped wave equation in which the values of the coefficients for a given porous medium are given as functions of its macroscopic parameters (as porosity, tortuosity and resistivity to the passage of air), as proposed by [28]. In particular, in Theorem 4 we propose a possible way to find the complex parameter in the Robin boundary condition of the former model that best approximates the latter. In Section 3, for the case of uniform Lipschitz boundaries satisfying a uniform $\varepsilon$-cone property for a fixed $\varepsilon>0$ and in addition having a uniform upper bound for their lengths inside non trivial balls (and hence which have boundaries with uniformly bounded lengths), we introduce the class of (shape) admissible domains, adding, as in the classical framework of shape optimization, the assumptions that all moving parts of the boundary belong to a compact set and that all domains having a fixed volume are included in a fixed bounded open set. In this class of admissible domains, for any fixed frequency we obtain the existence of an optimal shape in the sense that there exists a positive measure $\mu^{*}$ on the optimal shape $\Gamma$, equivalent to the usual Lebesgue measure $\lambda$, such that $\mu^{*} \geq \lambda$ and such that the weak solution of the corresponding Helmholtz problem realizes the infimum of the acoustical energy, the latter being defined using $\lambda$ on the boundary. In the case where $\mu^{*}=\lambda$ (this depends on the properties of the minimizing sequence which we don't know 
in advance), then it is also the minimum. Moreover, we notice that in order to have the existence of an optimal shape in a higher dimensional case (for instance in $\mathbb{R}^{3}$ or simply in $\mathbb{R}^{N}$ ) it is sufficient to replace everywhere the $N$-1-dimensional Lebesgue measure of the boundary by the $N$-1-dimensional Hausdorff measure, since in that case the Lebesgue measure of the $N$-1-dimensional boundary is not equal to the Hausdorff measure as for one dimensional curves, but proportional to it (see [22, Thrm. 1.12, p. 13], for the optimization in $\mathbb{R}^{3}$ the Lebesgue measure of the boundary is equal to $\pi / 4$ times the Hausdorff measure). See also Ref. [10] for a free discontinuity approach to a class of shape optimization problems involving a Robin condition on a free boundary.

To summarize, the rest of the paper is organized as follows. Section 2 introduces a damped acoustical propagation model in which damping occurs through the boundary. It is described by the Helmholtz problem with a Robin boundary condition with a complex coefficient $\alpha$, for which we give a well-posedness result on an admissible domain in $\mathbb{R}^{N}$ in the sense of Ref. [7]. The existence of an optimal shape in the introduced acoustical framework is proved in Section 3. We recall useful results from [7] in Appendix A. Appendix B details how to obtain the damping parameter $\alpha$ in the Robin boundary condition that best approximates a given model with dissipation in the volume.

\section{The model: motivation and known properties}

To describe the acoustic wave absorption by a porous medium, there are two possibilities. The first one is to consider wave propagation in two media, typically air and a wall, which corresponds to a damping in the volume. The most common mathematical model for this is the damped wave equation [8]. The second one is to consider only one lossless medium, air, and to model energy dissipation by a damping condition on the boundary. In both cases, we need to ensure the same order of energy damping corresponding to the physical characteristics of the chosen porous medium as its porosity $\phi$, tortuosity $\alpha_{h}$ and resistivity to the passage of air $\sigma$ [28].

Thanks to Ref. [28], we can define the coefficients in the damped wave equation (damping in volume) as functions of the above mentioned characteristics. More precisely, for a regular bounded domain $\Omega \subset \mathbb{R}^{2}$ (for instance $\partial \Omega \in C^{1}$ ) composed of two disjoint parts $\Omega=\Omega_{0} \cup \Omega_{1}$ of two homogeneous media, air in $\Omega_{0}$ and a porous material in $\Omega_{1}$, separated by an internal boundary $\Gamma$, we consider the following boundary value problem (for the pressure of the wave)

$$
\left\{\begin{array}{l}
\xi(x) \partial_{t}^{2} u+a(x) \partial_{t} u-\nabla \cdot(\eta(x) \nabla u)=0 \quad \text { in } \Omega \\
\left.\frac{\partial u}{\partial n}\right|_{\mathbb{R}_{t} \times \partial \Omega} \equiv 0, \quad[u]_{\Gamma}=[\eta \nabla u \cdot n]_{\Gamma}=0 \\
\left.u\right|_{t=0}=u_{0} \mathbb{1}_{\Omega_{0}},\left.\quad \partial_{t} u\right|_{t=0}=u_{1} \mathbb{1}_{\Omega_{0}}
\end{array}\right.
$$

with $\xi(x)=\frac{1}{c_{0}^{2}}, a(x)=0, \eta(x)=1$ in air, i.e., in $\Omega_{0}$, and

$$
\xi(x)=\frac{\phi \gamma_{p}}{c_{0}^{2}}, \quad a(x)=\sigma \frac{\phi^{2} \gamma_{p}}{c_{0}^{2} \rho_{0} \alpha_{h}}, \quad \eta(x)=\frac{\phi}{\alpha_{h}}
$$

in the porous medium, i.e., in $\Omega_{1}$. The external boundary $\partial \Omega$ is supposed to be rigid, i.e., Neumann boundary condition are applied, and on the internal boundary $\Gamma$ we have no-jump conditions on $u$ and $\eta \nabla u \cdot n$, where $n$ denotes the normal unit vector to $\Gamma$. Here, $c_{0}$ and $\rho_{0}$ denote respectively the sound velocity and the density of air, whereas $\gamma_{p}=7 / 5$ denotes the ratio of specific heats. But instead of energy absorption in volume, we can also consider the following frequency model of damping by the boundary. Let $\Omega$ be a connected bounded domain of $\mathbb{R}^{2}$ with a Lipschitz boundary $\partial \Omega$. We suppose that the boundary $\partial \Omega$ is divided into three parts $\partial \Omega=\Gamma_{D} \cup \Gamma_{N} \cup \Gamma$ (see Fig. 1 for an example of $\Omega$, chosen for the numerical calculations) and consider

$$
\begin{cases}\triangle u+\omega^{2} u=f(x), & x \in \Omega, \\ u=g(x) \quad \text { on } \Gamma_{D}, & \frac{\partial u}{\partial n}=0 \quad \text { on } \Gamma_{N}, \quad \frac{\partial u}{\partial n}+\alpha(x) u=\operatorname{Trh}(x) \quad \text { on } \Gamma,\end{cases}
$$


where $\alpha(x)$ is a complex-valued regular function with a strictly positive real part $(\operatorname{Re}(\alpha)>0)$ and a strictly negative imaginary part $(\operatorname{Im}(\alpha)<0)$.

Remark 1. This particular choice of the signs of the real and the imaginary parts of $\alpha$ are needed for the well-posedness properties [25] and the energy decay of the corresponding time-dependent problem. In addition, as the frequency $\omega>0$ is supposed to be fixed, $\alpha$ can contain a dependence on $\omega$, i.e., $\alpha \equiv \alpha(x, \omega)$.

Problem (2) is a frequency version of the following time-dependent wave propagation problem with $U(t, x)=e^{-i \omega t} u(x)$, considered in Ref. [9] for $g=0$ on $\Gamma_{D}$ :

$$
\begin{aligned}
& \partial_{t}^{2} U-\triangle U=-e^{-i \omega t} f(x), \\
& \left.U\right|_{t=0}=U_{0},\left.\quad \partial_{t} U\right|_{t=0}=U_{1}, \\
& \left.U\right|_{\Gamma_{D}}=g,\left.\quad \frac{\partial U}{\partial n}\right|_{\Gamma_{N}}=0, \\
& \frac{\partial U}{\partial n}-\frac{\operatorname{Im}(\alpha(x))}{\omega} \partial_{t} U+\left.\operatorname{Re}(\alpha(x)) U\right|_{\Gamma}=0 .
\end{aligned}
$$

To show the energy decay, we follow [9] and introduce the Hilbert space $X_{0}(\Omega)$, defined as the Cartesian product of the set of functions $u \in H^{1}(\Omega)$, which vanish on $\Gamma_{D}$ with the space $L_{2}(\Omega)$. The equivalent norm on $X_{0}(\Omega)$ is defined by

$$
\|(u, v)\|_{X_{0}(\Omega, \mu)}^{2}=\int_{\Omega}\left(\left|\nabla_{x} u\right|^{2}+|v|^{2}\right) \mathrm{d} x+\int_{\Gamma} \operatorname{Re}(\alpha(x))|u|^{2} \mathrm{~d} \mu
$$

with the corresponding inner product

$$
\left\langle\left(u_{1}, u_{2}\right),\left(v_{1}, v_{2}\right)\right\rangle=\int_{\Omega}\left(\nabla_{x} u_{1} \nabla_{x} v_{1}+u_{2} v_{2}\right) \mathrm{d} x+\int_{\Gamma} \operatorname{Re}(\alpha(x)) u_{1} v_{1} \mathrm{~d} \mu .
$$

Here $\mu$ is a Radon positive measure on $\Gamma$, which in the case of a regular $\Gamma$ (at least Lipschitz), if there are no specific assumptions, is equal to the Lebesgue measure on $\Gamma$, and in this case is denoted by $\lambda$.

The advantage of this norm is that the energy balance of the homogeneous problem (3)(6) has the form

$$
\partial_{t}\left(\left\|\left(U, \partial_{t} U\right)\right\|_{X_{0}(\Omega, \mu)}^{2}\right)=\frac{2}{\omega} \int_{\Gamma} \operatorname{Im}(\alpha(x))\left|\partial_{t} U\right|^{2} \mathrm{~d} \mu .
$$

Therefore, for $\operatorname{Im}(\alpha)<0$ on $\Gamma$, the energy decays in time. For the case of a smooth boundary $\partial \Omega$ (at least Lipschitz), we have the well-posedness of both models. Thanks to [9], for all $f \in L_{2}(\Omega),\left(U_{0}, U_{1}\right) \in X_{0}(\Omega)$ there exists a unique solution $\left(U, U_{t}\right) \in C(] 0, \infty\left[, X_{0}(\Omega)\right)$ of system (3)-(6) under the assumption that $\operatorname{Re}(\alpha(x))>0$ and $\operatorname{Im}(\alpha(x))<0$ are continuous functions.

For the frequency model (2) it is possible to generalize the weak well-posedness result in domains with Lipschitz boundaries [25] to domains with a more general class of boundaries, named Ahlfors $d$-regular sets or simply $d$-sets [30] (see Appendix A), using functional analysis tools on "admissible domains" developed in [7]. The interest of this generalization is that this class of domains is optimal in the sense that it is the largest possible class [7] which keeps the Sobolev extension operators, for instance $H^{1}(\Omega)$ to $H^{1}\left(\mathbb{R}^{N}\right)$, continuous. In what follows, for our well-posedness result we take $\Omega \subset \mathbb{R}^{N}, N \geq 2$.

We use, as in Refs. [7, 40], the existence of a $d$-dimensional $(0<d \leq N, d \in \mathbb{R})$ measure $\mu$ equivalent or equal to the Hausdorff measure $m_{d}$ on $\partial \Omega$ (see Definition 1) and a generalization of the usual trace theorem [30] (see Appendix A) and the Green formula [33, 7] in the sense of the Besov space $B_{\beta}^{2,2}(\partial \Omega)$ with $\beta=1-\frac{N-d}{2}>0$ constructed on $\partial \Omega$ with the help of the measure $\mu$ (for the definition of the Besov spaces on $d$-sets see Ref. [30] p.135 
and Ref. [41]). Note that for $d=N-1$, one has $\beta=\frac{1}{2}$ and $B_{\frac{1}{2}}^{2,2}(\partial \Omega)=H^{\frac{1}{2}}(\partial \Omega)$ as usual in the case of a Lipschitz boundary. In what follows we write $L_{2}(\partial \Omega, \mu)$ to specify that the space is defined with respect to the measure $\mu$. Some main elements of functional analysis on $d$-sets are presented in Appendix A.

Moreover, we stress that once a measure $\mu$ is fixed on the boundary $\partial \Omega$, it modifies the meaning of the Green formula in the following sense: for all $u$ and $v$ from $H^{1}(\Omega)$ with $\Delta u \in L_{2}(\Omega)$ the normal derivative of $u$ is understood as the linear continuous functional on the Besov space $B_{\beta}^{2,2}(\partial \Omega)$ constructed by $\mu$ according to the definition

$$
\left\langle\frac{\partial u}{\partial \nu}, \operatorname{Tr} v\right\rangle_{\left(\left(B_{\beta}^{2,2}(\partial \Omega)\right)^{\prime}, B_{\beta}^{2,2}(\partial \Omega)\right)}:=\int_{\Omega} v \Delta u \mathrm{~d} x+\int_{\Omega} \nabla v \cdot \nabla u \mathrm{~d} x .
$$

Considering the Helmholtz problem (2), we introduce the Hilbert space

$$
V(\Omega)=\left\{u \in H^{1}(\Omega) \mid u=0 \text { on } \Gamma_{D}\right\}
$$

equipped with the norm (equivalent to the usual norm $\|\cdot\|_{H^{1}(\Omega)}$ ) thanks to Theorem 3

$$
\|u\|_{V(\Omega, \mu)}^{2}=\int_{\Omega}|\nabla u|^{2} \mathrm{~d} x+\int_{\Gamma} \operatorname{Re}(\alpha)|u|^{2} d \mu
$$

and obtain the following well-posedness result:

Theorem 1. Let $\Omega \subset \mathbb{R}^{N}$ be a bounded admissible domain with a compact d-set boundary $(N-2<d<N)$ with a d-measure $\mu$ in the sense of Theorem 3, $\partial \Omega=\Gamma_{D} \cup \Gamma_{N} \cup \Gamma$ and $\beta=1-(N-d) / 2>0$. Let $\Gamma_{D}$ be also a d-set with the same properties as $\partial \Omega$ itself. Let in addition $\operatorname{Re}(\alpha(x))>0, \operatorname{Im}(\alpha(x))<0$ be smooth functions (at least continuous) on $\Gamma$. Then for all $f \in L_{2}(\Omega), g \in B_{\beta}^{2,2}\left(\Gamma_{D}\right), h \in H^{1}(\Omega)$, and $\omega>0$ there exists a unique solution $u$ of the Helmholtz problem (2), such that $(u-\tilde{g}) \in V(\Omega)$ (where $\tilde{g}$ is a lifting in $H^{1}(\Omega)$ of the boundary data $g$ ) in the following sense: for all $v \in V(\Omega)$

$$
\int_{\Omega} \nabla u \cdot \nabla \bar{v} \mathrm{~d} x-\omega^{2} \int_{\Omega} u \bar{v} \mathrm{~d} x+\int_{\Gamma} \alpha \operatorname{Tr} u \operatorname{Tr} \bar{v} d \mu=-\int_{\Omega} f \bar{v} \mathrm{~d} x+\int_{\Gamma} \operatorname{Tr} h \operatorname{Tr} \bar{v} d \mu .
$$

Moreover, the solution of problem (2) $u \in H^{1}(\Omega)$, continuously depends on the data: there exists a constant $C>0$, depending only on $\alpha, \omega$ and on $C_{P}(\Omega)$, such that

$$
\|u\|_{H^{1}(\Omega)} \leq C\left(\|f\|_{L_{2}(\Omega)}+\|g\|_{B_{\beta}^{2,2}\left(\Gamma_{D}\right)}+\|h\|_{H^{1}(\Omega)}\right)
$$

where $C_{P}(\Omega)$ is the Poincaré constant associated to $\Omega$. In particular, taking $g=0$, for all fixed $\omega>0$ the operator

$$
B: L_{2}(\Omega) \times H^{1}(\Omega) \rightarrow V(\Omega), \text { defined by } B(f, h)=u
$$

with $u$, the weak solution of (9), is a linear compact operator.

In addition, if, for $m \in \mathbb{N}^{*}, \partial \Omega \in C^{m+2}$ (hence $\mu$ is an $N-1$-measure on $\partial \Omega$ ), $f \in$ $H^{m}(\Omega)$ and $g \in H^{m+\frac{3}{2}}\left(\Gamma_{D}\right)$, then the solution $u$ belongs to $H^{m+2}(\Omega)$.

Proof. Let us start with $g=0$ and in addition suppose that $\alpha$ is a constant (the generalization for $\alpha(x)$ is straightforward). By the linearity of the Helmholtz problem (2), we set $u=u^{f}+u^{h}$, where $u^{f}$ is the solution of the Helmholtz problem with $\operatorname{Tr} h=0$ on $\Gamma$ and $u^{h}$ is the solution of the Helmholtz problem with $f=0$.

When $h=0$, the variational formulation for $u^{f}$ becomes: for all $\phi \in V(\Omega)$

$$
\left(u^{f}, \phi\right)_{V(\Omega, \mu)}-\omega^{2}\left(u^{f}, \phi\right)_{L_{2}(\Omega)}+i \operatorname{Im} \alpha\left(\operatorname{Tr} u^{f}, \operatorname{Tr} \phi\right)_{L_{2}(\Gamma, \mu)}=-(f, \phi)_{L_{2}(\Omega)},
$$

where we have defined the following equivalent inner product on $V(\Omega)$ :

$$
\forall(v, w) \in V(\Omega) \times V(\Omega) \quad(v, w)_{V(\Omega, \mu)}=(\nabla v, \nabla w)_{L_{2}(\Omega)}+\operatorname{Re} \alpha(\operatorname{Tr} v, \operatorname{Tr} w)_{L_{2}(\Gamma, \mu)} .
$$


Hence, the Riesz representation Theorem ensures the existence of a linear bounded operator $A: L_{2}(\Omega) \rightarrow V(\Omega)$ such that for $v \in L_{2}(\Omega)$

$$
\forall \phi \in V(\Omega) \quad(v, \phi)_{L_{2}(\Omega)}=(A v, \phi)_{V(\Omega, \mu)}
$$

and in addition, by the Poincaré inequality,

$$
\begin{aligned}
\|A v\|_{V(\Omega, \mu)} & =\sup _{\|\phi\|_{V(\Omega, \mu)}=1}\left|(v, \phi)_{L_{2}(\Omega)}\right| \leq \sup _{\|\phi\|_{V(\Omega, \mu)}=1}\|v\|_{L_{2}(\Omega)}\|\phi\|_{L_{2}(\Omega)} \\
& \leq C_{P}(\Omega) \sup _{\|\phi\|_{V(\Omega, \mu)}=1}\|v\|_{L_{2}(\Omega)}\|\phi\|_{V(\Omega, \mu)}=C_{P}(\Omega)\|v\|_{L_{2}(\Omega)}
\end{aligned}
$$

ensuring that $\|A\| \leq C_{P}(\Omega)$.

In the same way, using the Riesz representation Theorem we also define a linear bounded operator $\hat{A}: L_{2}(\Gamma, \mu) \rightarrow V(\Omega)$ such that for $w \in L_{2}(\Gamma, \mu)$

$$
\forall \phi \in V(\Omega) \quad(w, \operatorname{Tr} \phi)_{L_{2}(\Gamma, \mu)}=(\hat{A} w, \phi)_{V(\Omega, \mu)} .
$$

Indeed, it is sufficient to notice that for a fixed $w \in L_{2}(\Gamma, \mu)$ the form $\ell: \phi \in V(\Omega) \mapsto \ell(\phi)=$ $(w, \operatorname{Tr} \phi)_{L_{2}(\Gamma, \mu)} \in \mathbb{C}$ is linear and continuous on $V(\Omega)$ :

$$
\left|(w, \operatorname{Tr} \phi)_{L_{2}(\Gamma, \mu)}\right| \leq\|w\|_{L_{2}(\Gamma, \mu)}\|\operatorname{Tr} \phi\|_{L_{2}(\Gamma, \mu)} \leq C\|\phi\|_{V(\Omega, \mu)},
$$

thanks to the continuity and the linearity of the trace from $V(\Omega)$ to $L_{2}(\Gamma, \mu)$. Moreover, $\|\hat{A}\| \leq C(\operatorname{Re}(\alpha))$ since

$$
\begin{aligned}
\|\hat{A} w\|_{V(\Omega, \mu)} & =\sup _{\|\phi\|_{V(\Omega, \mu)}=1}\left|(w, \operatorname{Tr} \phi)_{L_{2}(\Gamma, \mu)}\right| \leq \sup _{\|\phi\|_{V(\Omega, \mu)}=1}\|w\|_{L_{2}(\Gamma, \mu)}\|\operatorname{Tr} \phi\|_{L_{2}(\Gamma, \mu)} \\
& \leq C(\operatorname{Re}(\alpha)) \sup _{\|\phi\|_{V(\Omega, \mu)}=1}\|w\|_{L_{2}(\Gamma, \mu)}\|\phi\|_{V(\Omega, \mu)}=C(\operatorname{Re}(\alpha))\|w\|_{L_{2}(\Gamma, \mu)} .
\end{aligned}
$$

Thus, denoting by $S$ the compact embedding operator of $V(\Omega)$ in $L_{2}(\Omega)$ (by the Poincaré inequality it holds that $\|S\| \leq C_{P}(\Omega)$ ), the variational formulation can be rewritten in the following form:

$$
\forall \phi \in V(\Omega) \quad\left(\left(I d-\omega^{2} A \circ S+i \operatorname{Im} \alpha \hat{A} \circ \operatorname{Tr}\right) u^{f}, \phi\right)_{V(\Omega, \mu)}=(-A f, \phi)_{V(\Omega, \mu)} .
$$

Thanks to the compactness of the trace operator $\operatorname{Tr}: V(\Omega) \rightarrow L_{2}(\partial \Omega)[7]$ (with $\|\operatorname{Tr}\| \leq$ $C(\operatorname{Re}(\alpha)))$, the operator $T=A \circ S-i \frac{\operatorname{Im} \alpha}{\omega^{2}} \hat{A} \circ \operatorname{Tr}: V(\Omega) \rightarrow V(\Omega)$ is compact as a composition of continuous and compact operators (with $\|T\| \leq C\left(\omega, \alpha, C_{P}(\Omega)\right)$ ). Thanks to the Fredholm alternative, it is then sufficient to prove that for $(h, f)=(0,0)$, then the unique solution is $u=0$, and this will allow us to conclude to the well-posedness of (12). Setting $f=0$ in (12), choosing $\phi=u^{f}$ and separating real and imaginary parts of the equality, we first obtain that $\operatorname{Tr} u^{f}=0$ on $\Gamma$ (since $|\operatorname{Im} \alpha|>0$ ). By the Robin boundary condition on $\Gamma$, we then obtain that $\frac{\partial u}{\partial n}=0$ on $\Gamma$ (in the sense of a continuous linear functional on $B_{\beta}^{2,2}(\Gamma)$ ). Then, $u^{f}=0$ in $\Omega$ follows by the uniqueness of the solution to the Cauchy problem for $\Delta+\omega^{2} I d$ in the connected domain $\Omega$ with Cauchy data on $\Gamma$ (see for example [17, Theorems 1.1 and 1.2], which can be directly adapted to the case of a domain $\Omega$ with a $d$-set boundary satisfying the conditions of Theorem 1 thanks to Theorem 3). The operator $\left(I d-\omega^{2} T\right)^{-1}$ is thus well defined and is also a linear continuous operator, by the Fredholm alternative theorem. Thus, we obtain

$$
\left\|u^{f}\right\|_{V(\Omega, \mu)} \leq \frac{\|A\|}{\left\|I d-\omega^{2} T\right\|}\|f\|_{L_{2}(\Omega)} \leq C\left(\omega, \alpha, C_{P}(\Omega)\right)\|f\|_{L_{2}(\Omega)} .
$$

In the same way, when $f=0$, the solution $u^{h}$ in $V(\Omega)$ satisfies the following variational formulation:

$$
\forall \phi \in V(\Omega) \quad\left(\left(I d-\omega^{2} A \circ S+i \operatorname{Im} \alpha \hat{A} \circ \operatorname{Tr}\right) u^{f}, \phi\right)_{V(\Omega, \mu)}=(\hat{A} \circ \operatorname{Tr} h, \phi)_{V(\Omega, \mu)} .
$$


Hence, as previously, we have

$$
\left\|u^{h}\right\|_{V(\Omega, \mu)} \leq \frac{\|\hat{A}\|\|\operatorname{Tr}\|}{\left\|I d-\omega^{2} T\right\|}\|h\|_{H^{1}(\Omega, \mu)} \leq C\left(\omega, \alpha, C_{P}(\Omega)\right)\|h\|_{H^{1}(\Omega, \mu)} .
$$

Consequently, we have proved the well-posedness and estimate (10) for $g=0$. Hence, by the standard lifting procedure, we can for instance chose $\tilde{g} \in H^{1}(\Omega)$ as the unique weak solution of (2) with $\omega=f=0$, with the homogeneous Neumann boundary conditions on $\Gamma_{N}$ and $\Gamma$ and with $\left.\tilde{g}\right|_{\Gamma_{D}}=g$, to obtain the result with $g \neq 0$.

To prove that the regularity of the boundary improves the regularity of the solution, we follow the classical approach, explained for elliptic equations in [21] Theorem 5 p. 323.

The linearity and the continuity of $B$ are evident and equivalent to estimate (10). Let us prove that for any fixed $\omega>0, B$ is also compact (see also Ref. [7] for the real Robin boundary condition). Indeed, let $\left(f_{j}, h_{j}\right) \rightarrow(f, h)$ in $L_{2}(\Omega) \times H^{1}(\Omega)$. Taking for all $j \in \mathbb{N}$, $u_{j}=B\left(f_{j}, h_{j}\right)$ and $u=B(f, h)$, by the linearity and the continuity of $B$ it follows that $u_{j} \stackrel{V(\Omega)}{\longrightarrow} u$. Knowing in addition that $\operatorname{Tr}: V(\Omega) \rightarrow L_{2}(\Gamma, \mu)$ and the inclusion of $H^{1}(\Omega)$ in $L_{2}(\Omega)$ are compact (see Ref. [7]) we have that $\operatorname{Tr} u_{j} \rightarrow \operatorname{Tr} u$ in $L_{2}(\Gamma, \mu)$ and $u_{j} \rightarrow u$ in $L_{2}(\Omega)$. Choosing $v=u_{j}$ in the variational formulation (9) we find

$$
\left\|u_{j}\right\|_{V(\Omega, \mu)}^{2}=\omega^{2}\left\|u_{j}\right\|_{L_{2}(\Omega)}^{2}-i \int_{\Gamma} \operatorname{Im} \alpha\left|\operatorname{Tr} u_{j}\right|^{2} d \mu-\int_{\Omega} f_{j} \bar{u}_{j} \mathrm{~d} x+\int_{\Gamma} \operatorname{Tr} h_{j} \overline{\operatorname{Tr} u_{j}} d \mu,
$$

and hence,

$$
\begin{aligned}
& \lim _{j \rightarrow+\infty}\left\|u_{j}\right\|_{V(\Omega, \mu)}^{2}=\omega^{2}\|u\|_{L_{2}(\Omega)}^{2}-i \int_{\Gamma} \operatorname{Im} \alpha|\operatorname{Tr} u|^{2} d \mu-\int_{\Omega} f \bar{u} \mathrm{~d} x+\int_{\Gamma} \operatorname{Tr} h \overline{\operatorname{Tr} u} d \mu \\
&=\|u\|_{V(\Omega, \mu)}^{2} .
\end{aligned}
$$

Having both $u_{j} \rightarrow u$ in $V(\Omega)$ and $\left\|u_{j}\right\|_{V(\Omega, \mu)} \rightarrow\|u\|_{V(\Omega, \mu)}$ implies that $u_{j} \rightarrow u$ in $V(\Omega)$ and hence $B$ is compact. Since the norm $\|u\|_{V(\Omega, \mu)}^{2}$ on $V(\Omega)$ is equivalent [7] to the norm

$$
\|u\|_{J}^{2}=A\|u\|_{L_{2}(\Omega)}^{2}+B\|\nabla u\|_{L_{2}(\Omega)}^{2}+C\|u\|_{L_{2}(\Gamma, \mu)}^{2},
$$

the operator $B$ is also compact with respect to this norm.

In order to relate the model with a damping on the boundary and the model with a damping in the volume, we propose in Appendix B a new theorem to identify the parameter $\alpha$ in the Robin boundary condition (Theorem 4). This parameter provides the best approximation (in some error minimizing sense) of the latter model by the former, in the case of a flat boundary $\Gamma$.

\section{Shape design problem}

We consider the two dimensional shape design problem, which consists in optimizing the shape of $\Gamma$ with the Robin dissipative condition in order to minimize the acoustic energy of system (2). The boundaries with the Neumann and Dirichlet conditions $\Gamma_{D}$ and $\Gamma_{N}$ are supposed to be fixed.

We also define a fixed open set $D$ with a Lipschitz boundary which contains all domains $\Omega$. Actually, as only a part of the boundary (precisely $\Gamma$ ) changes its shape, we also impose that the changing part always lies inside of the closure of a fixed open set $G$ with a Lipschitz boundary: $\Gamma \subset \bar{G}$. The set $G$ forbids $\Gamma$ to be too close to $\Gamma_{D}$, making the idea of an acoustical wall more realistic.

To introduce the class of admissible domains, on which we minimize the acoustical energy of system (2), we define $\mathcal{L}$ ip as the class of all domains $\Omega \subset D$ for which 


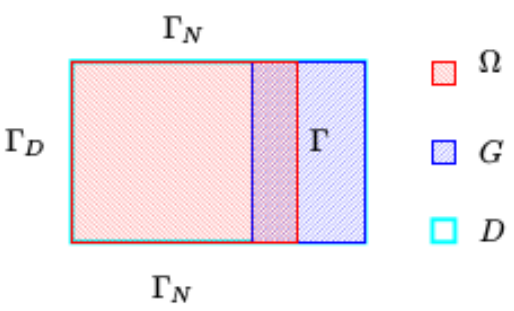

Figure 1: Example of a domain $\Omega$ in $\mathbb{R}^{2}$ with three types of boundaries: $\Gamma_{D}$ and $\Gamma_{N}$ are fixed and $\Gamma$ can be changed in the restricted area $\bar{G}$. Here $\Omega \cup G=D$ and obviously $\Omega \subset D$.

1. there exists a fixed $\varepsilon>0$ such that all domains $\Omega \in \mathcal{L}$ ip satisfy the $\varepsilon$-cone property [3, 14]: for all $x \in \partial \Omega$, there exists $\xi_{x} \in \mathbb{R}^{2}$ with $\left\|\xi_{x}\right\|=1$ such that for all $y \in \bar{\Omega} \cap B(x, \varepsilon)$

$$
C\left(y, \xi_{x}, \varepsilon\right)=\left\{z \in \mathbb{R}^{2} \mid\left(z-y, \xi_{x}\right) \geq \cos (\varepsilon)\|z-y\| \text { and } 0<\|z-y\|<\varepsilon\right\} \subset \Omega .
$$

2. there exists a fixed $\hat{c}>0$ such that for any $\Omega \in \mathcal{L}$ ip and for all $x \in \Gamma$ we have

$$
\int_{\Gamma \cap B(x, r)} d \lambda \leq \hat{c} r
$$

where $B(x, r)$ is the open Euclidean ball centered in $x$ with radius $r$ and $\lambda$ is the usual one-dimensional Lebesgue measure on $\Gamma$.

The uniform $\varepsilon$-cone property implies, by Remark 2.4 .8 [29, p. 55] and Theorem 2.4.7, that all boundaries of $\Omega \in \mathcal{L}$ ip are uniformly Lipschitz.

Let us notice that, by the boundedness of $D$ containing all $\Omega$, condition (14) implies that all $\Gamma$ for $\Omega \in \mathcal{L}$ ip have uniform length: there exists $M>0$ depending on the chosen $\hat{c}>0$ such that for all $\Omega \in \mathcal{L}$ ip it holds $\operatorname{Vol}(\partial \Omega)=\int_{\partial \Omega} d \lambda \leq M$.

The constant $M$ (and hence initially $\hat{c}$ ) can be chosen arbitrary large but finite. We denote by $\Omega_{0} \in \mathcal{L}$ ip and $\Gamma_{0} \subset \bar{G}$ the "reference" domain and the "reference" boundary respectively (actually $\partial \Omega_{0}=\Gamma_{D} \cup \Gamma_{N} \cup \Gamma_{0}$ ) corresponding to the initial shape before optimization.

Thus, the admissible class of domains can be defined as

$$
\begin{aligned}
& U_{a d}\left(\Omega_{0}, \varepsilon, \hat{c}, G\right)= \\
& \quad\left\{\Omega \in \mathcal{L} i p \mid \Gamma_{D} \cup \Gamma_{N} \subset \partial \Omega, \Gamma \subset \bar{G}, M_{0} \leq \int_{\Gamma} d \lambda \leq M(\hat{c}), \int_{\Omega} \mathrm{d} x=\operatorname{Vol}\left(\Omega_{0}\right)\right\},
\end{aligned}
$$

where $\hat{c}$ is given sufficiently large in the aim to have a sufficiently large constant $M>0$ in the sense that it is not less than $M_{0}>0$, which is the length of the straight line boundary. Moreover the case when $M$ is equal to the length of the plane boundary $M_{0}$ is the trivial case when $U_{a d}\left(\Omega_{0}, \varepsilon, \hat{c}, G\right)$ contains only one unique domain with the plane boundary, which hence is trivially optimal. Therefore the problem becomes interesting for a sufficiently large $M$.

In what follows we denote by $\lambda$ the 1-dimensional Lebesgue measure on the Lipschitz boundary $\Gamma$, by $m_{1}$ the 1-dimensional Hausdorff measure (which is equal to $\lambda$ on $\Gamma$ ) and we denote by $u(\Omega, \mu)$ the weak solution of the Helmholtz problem on $\Omega$ satisfying (9) with 1-dimensional Radon measure $\mu$.

We define

$$
J(\Omega, u(\Omega, \mu), \lambda)=A \int_{\Omega}|u(\Omega, \mu)|^{2} \mathrm{~d} x+B \int_{\Omega}|\nabla u(\Omega, \mu)|^{2} \mathrm{~d} x+C \int_{\Gamma}|u(\Omega, \mu)|^{2} d \lambda
$$


for given $\mu$ and $\lambda$ and with $A \geq 0, B \geq 0, C \geq 0$ positive constants for any fixed $\omega>0$.

Ideally we would like to minimize $J(\Omega, u(\Omega, \lambda), \lambda)$ on $U_{a d}\left(\Omega_{0}, \varepsilon, \hat{c}, G\right)$, however we are able to prove the existence of $\Omega_{o p t}$ in $U_{a d}\left(\Omega_{0}, \varepsilon, \hat{c}, G\right)$ with a 1-measure $\mu^{*}$, equivalent to $\lambda$, satisfying $\mu^{*}\left(\Gamma_{\text {opt }}\right) \geq \lambda\left(\Gamma_{\text {opt }}\right)$ on its boundary $\Gamma_{\text {opt }}$, such that $J\left(\Omega, u\left(\Omega, \mu^{*}\right), \lambda\right)$ realizes the infimum of $J(\Omega, u(\Omega, \lambda), \lambda)$ on $U_{a d}\left(\Omega_{0}, \varepsilon, \hat{c}, G\right)$. So, if $\mu^{*}\left(\Gamma_{\text {opt }}\right)=\lambda\left(\Gamma_{\text {opt }}\right)$, i.e. $\mu^{*}=\lambda$, then $\Omega_{\text {opt }}$ realizes the minimum of $J(\Omega, u(\Omega, \lambda), \lambda)$.

In order to keep the volume constraint, instead of Eq. (16) we can also consider the objective function

$$
\begin{aligned}
J_{1}(\Omega, u(\Omega, \lambda), \lambda)= & A \int_{\Omega}|u(\Omega, \lambda)|^{2} \mathrm{~d} x+B \int_{\Omega}|\nabla u(\Omega, \lambda)|^{2} \mathrm{~d} x+C \int_{\Gamma}|u(\Omega, \lambda)|^{2} d \lambda \\
& +\kappa\left(\operatorname{Vol}(\Omega)-\operatorname{Vol}\left(\Omega_{0}\right)\right)^{2},
\end{aligned}
$$

where $\kappa$ is some (large) positive constant penalizing the volume variation.

First of all let us prove the following lemma:

Lemma 1. Let $U_{a d}\left(\Omega_{0}, \varepsilon, \hat{c}, G\right)$ be the class of admissible domains defined in (15). Then the following statements hold:

1. $U_{a d}\left(\Omega_{0}, \varepsilon, \hat{c}, G\right)$ is closed with respect to the Hausdorff convergence, in the sense of characteristic functions in $L^{1}(D)$ and in the sense of compacts.

2. If $\left(\Omega_{n}\right)_{n \in \mathbb{N}^{*}} \subset U_{a d}\left(\Omega_{0}, \varepsilon, \hat{c}, G\right)$ then there exists a subsequence $\left(\Omega_{n_{k}}\right)_{k \in \mathbb{N}^{*}} \subset\left(\Omega_{n}\right)_{n \in \mathbb{N}^{*}}$ and a domain $\Omega \in U_{a d}\left(\Omega_{0}, \varepsilon, \hat{c}, G\right)$ such that $\left(\Omega_{n_{k}}\right)_{k \in \mathbb{N}^{*}}$ converges to $\Omega$ with respect to these three types of convergences and in addition $\bar{\Omega}_{n_{k}}$ and $\Gamma_{n_{k}}$ converge in the sense of Hausdorff respectively to $\bar{\Omega}$ and $\Gamma$.

3. Let $\left(\Omega_{n}\right)_{n \in \mathbb{N}^{*}} \subset U_{a d}\left(\Omega_{0}, \varepsilon, \hat{c}, G\right)$ be a sequence converging to $\Omega$ in $U_{a d}\left(\Omega_{0}, \varepsilon, \hat{c}, G\right)$ in the sense of point 2. Then there exists a subsequence $\left(\Omega_{n_{k}}\right)_{k \in \mathbb{N}^{*}}$ with boundaries $\left(\Gamma_{n_{k}}\right)_{k \in \mathbb{N}^{*}}$ and there exists a positive Radon 1-measure $\mu^{*}$ with support on $\Gamma$, equivalent to the one-dimensional Hausdorff measure on it or simply to its Lebesgue measure $\lambda$, such that

(a) $\forall \psi \in C(\bar{D}) \quad \int_{\Gamma_{n_{k}}} \psi d \lambda \rightarrow \int_{\Gamma} \psi d \mu^{*}$,

(b) $\int_{\Gamma} d \lambda \leq \int_{\Gamma} d \mu^{*}$ or equivalently $\mu^{*}(\Gamma) \geq \lambda(\Gamma)$.

Proof. Point 1. Let us denote by

$U=\left\{\right.$ domains $\Omega \subset D \mid \Omega$ satisfies the $\varepsilon-$ cone property and $\left.\Gamma_{D} \cup \Gamma_{N} \subset \partial \Omega, \Gamma \subset \bar{G}\right\}$,

which obviously contains $U_{a d}\left(\Omega_{0}, \varepsilon, \hat{c}, G\right)$, then thanks to [29] Theorem 2.4 .10 p. 56 (see also p. 145), the set $U$ is closed with respect to the Hausdorff convergence (i.e. if $\left(\Omega_{n}\right)_{n \in \mathbb{N}^{*}} \subset$ $U_{a d}\left(\Omega_{0}, \varepsilon, \hat{c}, G\right)$ and $d_{H}\left(D \backslash \Omega_{n}, D \backslash \Omega\right) \rightarrow 0$ for $n \rightarrow+\infty$, which means that $\Omega_{n} \rightarrow \Omega$ in the sense of Hausdorff, then $\Omega \in U$ ), but also in the sense of characteristic functions, (i.e. if $\left(\Omega_{n}\right)_{n \in \mathbb{N}^{*}} \subset U_{a d}\left(\Omega_{0}, \varepsilon, \hat{c}, G\right)$ and $\mathbb{1}_{\Omega_{n}} \rightarrow \mathbb{1}_{\Omega}$ for $n \rightarrow+\infty$ in $L_{l o c}^{p}\left(\mathbb{R}^{2}\right)$ for all $p \in[1, \infty[$, then $\Omega \in U$ ) and also in the sense of compacts (if for all $K$ compact in $\Omega$ it follows that $K \subset \Omega_{n}$ and for all $O$ compact in $D \backslash \bar{\Omega}$ it follows that $O \in D \backslash \bar{\Omega}_{n}$ for a sufficiently large $n$, then $\Omega \in U)$. Consequently, if $\left(\Omega_{n}\right)_{n \in \mathbb{N}^{*}} \subset U_{a d}\left(\Omega_{0}, \varepsilon, \hat{c}, G\right) \subset U$ converges in these three senses to $\Omega \in U$, then, since for all $n$ the domains $\Omega_{n}$ are subsets of a fixed open set $D$ which is bounded in $\mathbb{R}^{2}$, the sequence $\mathbb{1}_{\Omega_{n}} \rightarrow \mathbb{1}_{\Omega}$ for $n \rightarrow+\infty$ in $L^{1}(D)$. This strong convergence of the characteristic functions [29, Prop. 2.3.6, p.47] implies that the perimeter and volume are respectively lower semicontinuous and continuous functions of $\Omega_{n}$. Hence,

$$
\operatorname{Vol}(\Omega)=\lim _{n \rightarrow+\infty} \operatorname{Vol}\left(\Omega_{n}\right)=\operatorname{Vol}\left(\Omega_{0}\right)
$$


and in addition, since $\Gamma_{N}$ and $\Gamma_{D}$ are fixed and all $\Gamma_{n} \subset \bar{G}$,

$$
\int_{\Gamma} d \lambda \leq \liminf _{n \rightarrow+\infty} \int_{\Gamma_{n}} d \lambda
$$

In particular Eq. (18) ensures that as soon as for all $\Gamma_{n}$, (14) holds with $\hat{c}>0$ independent of $n$, then it holds also for $\Gamma$ and in the same way $\int_{\Gamma} d \lambda \leq M$. As $\Gamma$ is Lipschitz and in particular is given by a continuous curve, we also have $\int_{\Gamma} d \lambda \geq M_{0}$. Thus, we conclude that $U_{a d}\left(\Omega_{0}, \varepsilon, \hat{c}, G\right)$ is closed.

Point 2. For the second statement it is sufficient to notice that Theorem 2.4.10 [29, p. 56] holds on $U$. As $U$ is compact with respect to the three considered convergences and as $U_{a d}\left(\Omega_{0}, \varepsilon, \hat{c}, G\right)$ is its closed subset, then it is compact too.

Point 3. Let us prove the third point. We take a sequence $\left(\Omega_{n}\right)_{n \in \mathbb{N}^{*}}$ in $U_{a d}\left(\Omega_{0}, \varepsilon, \hat{c}, G\right)$ which converges to $\Omega \in U_{a d}\left(\Omega_{0}, \varepsilon, \hat{c}, G\right)$ in all senses of point 2. By Lemma 3.5 [22] the Lipschitz curves are 1-sets (see Definition 1). We take the 1-dimensional Hausdorff measures $\eta_{n}$ on $D$ in $\mathbb{R}^{2}$ having their supports on $\Gamma_{n}$. The sequence of measures $\left(\eta_{n}\right)_{n \in \mathbb{N}^{*}} \subset$ $\mathcal{M}_{b}(D)$ is uniformly bounded on $D$ (for all $n\left\|\eta_{n}\right\|_{1}=\eta_{n}(D)=\int_{\Gamma_{n}} d \lambda \leq M$ ). Then by [5, Thm. 1.59, p 26] or [29, Prop. 2.3.9,p. 48] there exits a weakly* convergent subsequence and there exists a Radon positive measure $\mu^{*} \in \mathcal{M}_{b}(D)$ such that $\eta_{n_{k}}$ converges weakly* to $\mu^{*}$

$$
\forall \psi \in C_{0}(D) \quad \int_{D} \psi d \eta_{n_{k}}=\int_{\Gamma_{n_{k}}} \psi d \lambda \rightarrow \int_{D} \psi d \mu^{*}
$$

As $\Gamma_{n_{k}}$ converges in the sense of Hausdorff to $\Gamma$ then the support of $\mu^{*}$ is equal to $\Gamma$ : $\int_{D} \psi d \mu^{*}=\int_{\Gamma} \psi d \mu^{*}$, and hence

$$
\forall \psi \in C_{0}(D) \quad \int_{\Gamma_{n_{k}}} \psi d \lambda \rightarrow \int_{\Gamma} \psi d \mu^{*}
$$

But the restriction of $\mu^{*}$ to $\Gamma$ is not necessarily equal to the Hausdorff measure on $\Gamma$, we only can prove that it is an equivalent 1-dimensional measure to the usual Lebesgue measure on $\Gamma$ by [30, Prop. 1, p. 30] (see also Theorem 1 on p. 32). Indeed, taking in the definition of the weak* limit a particular $\psi$ equal to 1 for all $x \in \bar{G}$ and knowing that all $\Gamma_{n_{k}}$ and $\Gamma$ itself are subsets of $\bar{G}$, we find by the lower semicontinuity of perimeters that

$$
\int_{\Gamma} d \mu^{*}=\lim _{k} \int_{\Gamma_{n_{k}}} d \lambda=\liminf _{k} \int_{\Gamma_{n_{k}}} d \lambda \geq \int_{\Gamma} d \lambda
$$

For instance, if $\Gamma_{n}$ are boundaries with a constant length which oscillate around a plane segment that has a length twice smaller than $\Gamma_{n}$, and such that $\Gamma_{n} \rightarrow \Gamma$ in the sense of Hausdorff, it easy to see that

$$
\lambda\left(\Gamma_{n}\right) \rightarrow \mu^{*}(\Gamma)=2 \lambda(\Gamma)>\lambda(\Gamma)
$$

In addition, once again by the definition of $G$ containing all $\Gamma_{n}$ and $\Gamma$ in $D$, the weak* limit (19) also holds for all $\psi \in C(\bar{D})$. Consequently, if $\eta$ is the 1-dimensional Hausdorff measure with support equal to $\Gamma$, then $\mu^{*} \geq \eta$ and thus there exists $c_{1}>0$ such that

$$
c_{1} r \leq \eta(B(x, r)) \leq \mu^{*}(B(x, r)) \quad x \in \Gamma, 0<r \leq 1 .
$$

To show that $\mu^{*}$ is also a 1-measure we need to have the upper bound too. Since $B(x, r)$ is open, it holds, thanks to (14) and to the uniform bound of lengths $M_{0} \leq \eta_{n_{k}}\left(\Gamma_{n_{k}}\right)=$ $\lambda\left(\Gamma_{n_{k}}\right) \leq M$,

$$
\frac{1}{\mu^{*}(\Gamma)} \mu^{*}(B(x, r)) \leq \liminf _{k} \frac{1}{\int_{\Gamma_{n_{k}}} d \lambda} \eta_{n_{k}}(B(x, r)) \leq \frac{1}{M_{0}} \liminf _{k} \eta_{n_{k}}(B(x, r)) \leq \frac{1}{M_{0}} \hat{c} r
$$


by the Portmanteau theorem (see for instance Theorem 11.1.1 in Dudley [18]). Hence, $\mu^{*}(B(x, r)) \leq c_{2} r$ with the constant $c_{2}=\frac{\mu^{*}(\Gamma)}{M_{0}} \hat{c}>0$ independent of $n_{k}$.

Then we conclude that $\mu^{*}$ is 1-measure on $\Gamma$ and thus equivalent to $\eta$ by [30, Prop. 1,p. 30].

Let us now prove the existence of an optimal shape in a certain sense:

Theorem 2. Let $\Omega_{0} \subset D$ be a domain of the class $\mathcal{L}$ ip with a Lipschitz boundary $\partial \Omega_{0}$ of bounded length, such that $\Gamma_{D} \cup \Gamma_{N} \subset \partial \Omega_{0}$ and $\Gamma_{0}=\partial \Omega_{0} \backslash\left(\Gamma_{D} \cup \Gamma_{N}\right) \subset \bar{G}, U_{a d}$ be defined by (15) and $\omega>0$ be fixed. For the objective function $J(\Omega, u(\Omega, \lambda), \lambda)$, defined in (16) and constructed with the weak solution of the Helmholtz problem (2) for some fixed $\alpha \in C(\bar{G})$, $f \in L_{2}(D), g \in H^{\frac{1}{2}}\left(\Gamma_{D}\right)$ and $h \in H^{1}(D)$, there exists $\Omega_{o p t} \in U_{a d}\left(\Omega_{0}, \varepsilon, \hat{c}, G\right)$ and there exists a finite valued 1-dimensional positive measure $\mu^{*}$ on its boundary $\Gamma_{\text {opt }}$ equivalent to $\lambda$ such that

$$
\int_{\Gamma_{o p t}} d \mu^{*} \geq \int_{\Gamma_{o p t}} d \lambda
$$

and

$$
J\left(\Omega_{o p t}, u\left(\Omega_{o p t}, \mu^{*}\right), \lambda\right) \leq \inf _{\Omega \in U_{a d}\left(\Omega_{0}, \varepsilon, \hat{c}, G\right)} J(\Omega, u(\Omega, \lambda), \lambda)=J\left(\Omega_{o p t}, u\left(\Omega_{o p t}, \mu^{*}\right), \mu^{*}\right) .
$$

If $\mu^{*}=\lambda$, then $J\left(\Omega_{o p t}, u\left(\Omega_{o p t}, \lambda\right), \lambda\right)$ is the minimum on $U_{a d}\left(\Omega_{0}, \varepsilon, \hat{c}, G\right)$.

Proof. Let us start by noticing that, since all $\Omega \in U_{a d}\left(\Omega_{0}, \varepsilon, \hat{c}, G\right)$ are included in the same domain $D$, it follows that the Poincaré constants in Theorem 1 can all be bounded by the same constant $C(\operatorname{Vol}(D))$ depending only on the volume of $D$.

Let $\left(\Omega_{n}\right)_{n \in \mathbb{N}^{*}} \subset U_{a d}\left(\Omega_{0}, \varepsilon, \hat{c}, G\right)$ be a minimizing sequence of the functional $J(\Omega)$ (this minimizing sequence exists since $J(\Omega) \geq 0$ ).

Hence, by Lemma 1 there exist a domain $\Omega$ with $\Gamma$ and a subsequence $\left(\Omega_{n_{k}}\right)_{k \in \mathbb{N}}$ converging in these three senses to $\Omega$ and such that $\bar{\Omega}_{n_{k}}$ and $\Gamma_{n_{k}}$ converge in the sense of Hausdorff to $\bar{\Omega}$ and $\Gamma$ respectively (all other boundary parts are the same as the sequence is in $\left.U_{a d}\left(\Omega_{0}, \varepsilon, \hat{c}, G\right)\right)$. In the aim to abbreviate the notations, in what follows the index $n_{k}$ is changed to $n$.

By point 3 of Lemma 1 there exist a subsequence of boundaries $\left(\Gamma_{n_{k}}\right)_{k \in \mathbb{N}^{*}} \subset\left(\Gamma_{n}\right)_{n \in \mathbb{N}^{*}}$ and a positive measure $\mu^{*}$ on $\Gamma$, equivalent to $\lambda$, such that Eq. (20) holds and

$$
\forall \psi \in C(\bar{D}) \quad \int_{\Gamma_{n_{k}}} \psi d \lambda \rightarrow \int_{\Gamma} \psi d \mu^{*} .
$$

Therefore, all norms of $L^{2}(\Gamma)$ and $V(\Omega)$ computed with this measure on $\Gamma$ are equivalent to the corresponding norms computed with the Lebesgue measure on $\Gamma$.

To avoid complicated notations we denote again $\Gamma_{n_{k}}$ by $\Gamma_{n}$.

Let us consider now the solutions $\left(u_{n}\right)_{n \in \mathbb{N}^{*}}$ of the Helmholtz problem on $\left(\Omega_{n}\right)_{n \in \mathbb{N}^{*}}$.

Since domains of $\left.U_{a d}\left(\Omega_{0}, \varepsilon, \hat{c}, G\right)\right)$ have Lipschitz boundaries with finite perimeters, we use (see [14]) the fact that the norm of the extension operator $E: H^{1}(\Omega) \rightarrow H^{1}\left(\mathbb{R}^{2}\right)$ is bounded on such a family of domains $i . e$ there exists a constant $C_{E}>0$ independent of $n$ such that

$$
\left\|\left.E u_{n}\right|_{D}\right\|_{H^{1}(D)} \leq C_{E}\left\|u_{n}\right\|_{H^{1}\left(\Omega_{n}\right)},
$$

to deduce that the sequence $\left(\left.E u_{n}\right|_{D}\right)_{n \in \mathbb{N}^{*}}$ is bounded in $H^{1}(D)$ :

$$
\begin{aligned}
\left\|\left.E u_{n}\right|_{D}\right\|_{H^{1}(D)} & \leq C_{E}\left\|u_{n}\right\|_{H^{1}\left(\Omega_{n}\right)} \\
& \leq C_{E} C\left(\alpha, \omega, C_{p}\left(\Omega_{n}\right)\right)\left(\|f\|_{L_{2}\left(\Omega_{n}\right)}+\|g\|_{H^{\frac{1}{2}\left(\Gamma_{D}\right)}}+\|h\|_{H^{1}\left(\Omega_{n}\right)}\right) \\
& \leq C_{E} C(\alpha, \omega, \operatorname{Vol}(D))\left(\|f\|_{L_{2}(D)}+\|g\|_{H^{\frac{1}{2}\left(\Gamma_{D}\right)}}+C_{E}\|h\|_{H^{1}(D)}\right),
\end{aligned}
$$


which means that there exists a constant $C>0$ independent of $n$ such that

$$
\left\|\left.E u_{n}\right|_{D}\right\|_{H^{1}(D)} \leq C \text { for all } n \text {. }
$$

Here, in addition to the uniform boundedness of the extension operators, we have also used Eq. (10) and the fact that $\Gamma_{D}$ is the same for all $n$. Consequently, there exists $u^{*} \in$ $H^{1}(D)$ such that $\left.E u_{n}\right|_{D} \rightarrow u^{*}$ in $H^{1}(D)$. By compactness of the trace operator $\operatorname{Tr}_{\Gamma}$ : $H^{1}(D) \rightarrow L_{2}(\Gamma)$ and of the inclusion of $H^{1}(D)$ in $L_{2}(D)$ (see Theorem 3), we directly have that $\operatorname{Tr}_{\Gamma}\left(\left.E u_{n}\right|_{D}\right) \rightarrow \operatorname{Tr}_{\Gamma}\left(u^{*}\right)$ in $L_{2}(\Gamma)$ and $\left.E u_{n}\right|_{D} \rightarrow u^{*}$ in $L_{2}(D)$.

Let us show that $u^{*}$ is equal to the weak solution $u\left(\Omega, \mu^{*}\right)$ of $(9)$ on $\Omega$ in the sense of the variational formulation (9) considered with $\mu^{*}$.

From the variational formulation (9), taking $f \in L_{2}(D)$ and $h \in H^{1}(D)$, let us consider linear functionals defined for a fixed $v \in V(D)$ and for all $w_{n}$ and $w$ in $V(D)$

$$
\begin{aligned}
F^{n}\left[w_{n}, v\right]= & \left(\nabla w_{n}, \nabla v\right)_{L_{2}\left(\Omega_{n}\right)}-\omega^{2}\left(w_{n}, v\right)_{L_{2}\left(\Omega_{n}\right)}+\left(\alpha w_{n}, v\right)_{L_{2}\left(\Gamma_{n}, \lambda\right)} \\
& +(f, v)_{L_{2}\left(\Omega_{n}\right)}-\left(\operatorname{Tr}_{\Gamma_{n}} h, v\right)_{L_{2}\left(\Gamma_{n}, \lambda\right)}, \\
F[w, v]= & (\nabla w, \nabla v)_{L_{2}(\Omega)}-\omega^{2}(w, v)_{L_{2}(\Omega)}+(\alpha w, v)_{L_{2}\left(\Gamma, \mu^{*}\right)} \\
& +(f, v)_{L_{2}(\Omega)}-\left(\operatorname{Tr}_{\Gamma} h, v\right)_{L_{2}\left(\Gamma, \mu^{*}\right)} .
\end{aligned}
$$

We start by showing that as soon as $w_{n} \rightarrow w$ in $V(D)$

$$
\forall v \in V(D) \quad F^{n}\left[w_{n}, v\right] \rightarrow F[w, v] \text { for } n \rightarrow+\infty .
$$

Thus we consider

$$
\begin{aligned}
\left|F^{n}\left[w_{n}, v\right]-F[w, v]\right| & \left|\left(\nabla w_{n}, \mathbb{1}_{\Omega_{n}} \nabla v\right)_{L_{2}(D)}-\left(\nabla w, \mathbb{1}_{\Omega} \nabla v\right)_{L_{2}(D)}\right| \\
& +\omega^{2}\left|\left(w_{n}, \mathbb{1}_{\Omega_{n}} v\right)_{L_{2}(D)}-\left(w, \mathbb{1}_{\Omega} v\right)_{L_{2}(D)}\right| \\
& +\left|\left(\alpha w_{n}, v\right)_{L_{2}\left(\Gamma_{n}, \lambda\right)}-(\alpha w, v)_{L_{2}\left(\Gamma, \mu^{*}\right)}\right|+\left|\left(f,\left(\mathbb{1}_{\Omega_{n}}-\mathbb{1}_{\Omega}\right) v\right)_{L_{2}(D)}\right| \\
& +\left|\left(\operatorname{Tr}_{\Gamma_{n}} h, v\right)_{L_{2}\left(\Gamma_{n}, \lambda\right)}-\left(\operatorname{Tr}_{\Gamma} h, v\right)_{L_{2}\left(\Gamma, \mu^{*}\right)}\right| .
\end{aligned}
$$

Since $\Omega_{n} \rightarrow \Omega$ in the sense of characteristic functions and $v \in H^{1}(D)$, we directly have that $\mathbb{1}_{\Omega_{n}} \nabla v \rightarrow \mathbb{1}_{\Omega} \nabla v$ in $L_{2}(D)$, which with $w_{n} \rightarrow w$ in $V(D)$ gives that

$$
\left(\nabla w_{n}, \mathbb{1}_{\Omega_{n}} \nabla v\right)_{L_{2}(D)} \rightarrow\left(\nabla w, \mathbb{1}_{\Omega} \nabla v\right)_{L_{2}(D)} \text { for } n \rightarrow+\infty .
$$

By the compactness of the inclusion of $H^{1}(D)$ in $L_{2}(D), w_{n} \rightarrow w$ in $L_{2}(D)$ and by the convergence of the characteristic functions $\mathbb{1}_{\Omega_{n}} v \rightarrow \mathbb{1}_{\Omega} v$ in $L_{2}(D)$, hence we also have

$$
\left(w_{n}, \mathbb{1}_{\Omega_{n}} v\right)_{L_{2}(D)} \rightarrow\left(w, \mathbb{1}_{\Omega} v\right)_{L_{2}(D)}
$$

and similarly, $\left(f,\left(\mathbb{1}_{\Omega_{n}}-\mathbb{1}_{\Omega}\right) v\right)_{L_{2}(D)} \rightarrow 0$.

Let us prove that

$$
\forall v \in C(\bar{D}) \cap V(D) \quad\left(\alpha w_{n}, v\right)_{L_{2}\left(\Gamma_{n}, \lambda\right)} \rightarrow(\alpha w, v)_{L_{2}\left(\Gamma, \mu^{*}\right)} .
$$

Thanks to [36] Theorem 1.1.6/2, for all domains $\Omega \in U_{a d}\left(\Omega_{0}, \varepsilon, \hat{c}, G\right)$ and $D$ itself, the space $C^{\infty}(\bar{\Omega}) \cap V(\Omega)$ is dense in $V(\Omega)$. Thus there exists a sequence $\left(\phi_{m}\right)_{m \in \mathbb{N}} \subset C^{\infty}(\bar{D}) \cap$ $V(D)$ converging strongly to $w \in V(D)$. Therefore, following [12] we have

$$
\begin{aligned}
& \left|\int_{\Gamma_{n}} \operatorname{Tr} w_{n} \operatorname{Tr} v d \lambda-\int_{\Gamma} \operatorname{Tr} w \operatorname{Tr} v d \mu^{*}\right| \leq \\
& \leq\left|\int_{\Gamma_{n}}\right| \operatorname{Tr} w_{n}-\operatorname{Tr} w|| \operatorname{Tr} v|d \lambda|+\left|\int_{\Gamma_{n}}\right| \operatorname{Tr} w-\operatorname{Tr} \phi_{m}|| \operatorname{Tr} v|d \lambda| \\
& +\left|\int_{\Gamma_{n}} \operatorname{Tr} \phi_{m} \operatorname{Tr} v d \lambda-\int_{\Gamma} \operatorname{Tr} \phi_{m} \operatorname{Tr} v d \mu^{*}\right|+\left|\int_{\Gamma}\right| \operatorname{Tr} \phi_{m}-\operatorname{Tr} w|| \operatorname{Tr} v\left|d \mu^{*}\right| .
\end{aligned}
$$


We start by estimating the first term in Eq. (26): control it with the Cauchy-Schwarz inequality

$$
\left|\int_{\Gamma_{n}}\right| \operatorname{Tr} w_{n}-\operatorname{Tr} w|| \operatorname{Tr} v|d \lambda| \leq\left\|\operatorname{Tr}\left(w_{n}-w\right)\right\|_{L_{2}\left(\Gamma_{n}, \lambda\right)}\|\operatorname{Tr} v\|_{L_{2}\left(\Gamma_{n}, \lambda\right)} .
$$

Moreover, there exists a positive constant $C_{\sigma}>0$ independent of $n$ such that for $\frac{1}{2}<\sigma \leq 1$ it holds

$$
\forall w \in H^{\sigma}\left(\mathbb{R}^{2}\right) \quad\left\|\operatorname{Tr}_{\Gamma_{n}} w\right\|_{L_{2}\left(\Gamma_{n}, \lambda\right)}^{2} \leq C_{\sigma}\|w\|_{H^{\sigma}\left(\mathbb{R}^{2}\right)}^{2} .
$$

It is a direct corollary of the proof of [12] Theorem 5.3 and the fact that the lengths of $\Gamma$ and all $\Gamma_{n}$ are finite and bounded by a constant, denoted by $M$. In addition,

$$
\|\operatorname{Tr} v\|_{L_{2}\left(\Gamma_{n}, \lambda\right)} \leq \lambda\left(\Gamma_{n}\right)\|v\|_{L^{\infty}(D)} \leq M\|v\|_{L^{\infty}(D)} .
$$

Moreover, by Theorem 5.8 [12], for $D$ (but also for all domains in $U_{a d}\left(\Omega_{0}, \varepsilon, \hat{c}, G\right)$ ) there exists a bounded linear extension operator $E_{\sigma}: H^{\sigma}(D) \rightarrow H^{\sigma}\left(\mathbb{R}^{2}\right), \frac{1}{2}<\sigma \leq 1$, with

$$
\left\|E_{\sigma} v\right\|_{H^{\sigma}\left(\mathbb{R}^{2}\right)} \leq C_{D}\|v\|_{H^{\sigma}(D)} .
$$

Hence, applying Eq. (27) and Eq. (28), we obtain that

$$
\left\|\operatorname{Tr}\left(w_{n}-w\right)\right\|_{L_{2}\left(\Gamma_{n}, \lambda\right)} \leq C_{\sigma}\left\|E_{\sigma}\left(w_{n}-w\right)\right\|_{H^{\sigma}\left(\mathbb{R}^{2}\right)} \leq C_{\sigma} C_{D}\left\|w_{n}-w\right\|_{H^{\sigma}(D)},
$$

from where, by the compactness of the embedding of $H^{1}(D)$ in $H^{\sigma}(D)$ for $\frac{1}{2}<\sigma<1$, we finally have that $\left\|w_{n}-w\right\|_{H^{\sigma}(D)} \rightarrow 0$ for $n \rightarrow+\infty$ and consequently the first term in Eq. (26) converges to 0 for $n \rightarrow+\infty$.

For the second term (and in the same way the last term) in Eq. (26), as previously we directly find

$$
\left|\int_{\Gamma_{n}}\right| \operatorname{Tr} w-\operatorname{Tr} \phi_{m}\|\operatorname{Tr} v|d \lambda| \leq C\| w-\phi_{m} \|_{H^{1}(D)} \rightarrow 0 \text { for } m \rightarrow+\infty
$$

with a constant $C>0$ independent of $n$. For the last term we simply replace $\Gamma_{n}$ by $\Gamma$, knowing that $\Omega \in U_{a d}\left(\Omega_{0}, \varepsilon, \hat{c}, G\right)$. Hence, for all $\varepsilon>0$ there exists $k \in \mathbb{N}$ (uniformly on $n$ ) such that

$$
\forall m \geq k \quad \max \left\{\left|\int_{\Gamma_{n}}\right| \operatorname{Tr} w-\operatorname{Tr} \phi_{m}|| \operatorname{Tr} v|d \lambda|,\left|\int_{\Gamma}\right| \operatorname{Tr} \phi_{m}-\operatorname{Tr} w|| \operatorname{Tr} v\left|d \mu^{*}\right|\right\}<\varepsilon .
$$

Thus, let us fix such an $m$.

Finally, for the third term in Eq. (26), we use Eq. (22) which, by the continuity and the boundedness of $\phi_{m} v$ in $\bar{D}$ with a standard density argument, implies

$$
\int_{\Gamma_{n}}\left|\operatorname{Tr} \phi_{m} \operatorname{Tr} v\right| d \lambda \rightarrow \int_{\Gamma}\left|\operatorname{Tr} \phi_{m} \operatorname{Tr} v\right| d \mu^{*} \text { for } n \rightarrow+\infty .
$$

Therefore, for the sufficiently large $m$ that we have fixed, we also have that

$$
\forall \varepsilon>0 \exists p \in \mathbb{N}: \forall n \geq p\left|\int_{\Gamma_{n}}\right| \operatorname{Tr} \phi_{m} \operatorname{Tr} v\left|d \lambda-\int_{\Gamma}\right| \operatorname{Tr} \phi_{m} \operatorname{Tr} v\left|d \mu^{*}\right|<\varepsilon .
$$

Putting all results together for the four terms of Eq. (26), we obtain Eq. (25), which by the density of $C(\bar{D}) \cap V(D)$ in $V(D)$, also holds for all $v \in V(D)$. Consequently, we also have, as $h \in V(D)$

$$
\forall v \in V(D) \quad\left(\operatorname{Tr}_{\Gamma_{n}} h, v\right)_{L_{2}\left(\Gamma_{n}, \lambda\right)} \rightarrow\left(\operatorname{Tr}_{\Gamma} h, v\right)_{L_{2}\left(\Gamma, \mu^{*}\right)} \quad \text { for } n \rightarrow+\infty .
$$

This concludes the proof of Eq. (24). 
Hence, taking $w_{n}=\left.E u_{n}\right|_{D} \in V(D)$, i.e. the extensions of solutions on $\Omega_{n}$, which are uniformly bounded and weakly converge to $u^{*} \in V(D)$, we find that for all $v \in V(D)$

$$
0=F^{n}\left[\left.E u_{n}\right|_{D}, v\right] \rightarrow F\left[u^{*}, v\right]=0 \text { for } n \rightarrow+\infty .
$$

This means that $u^{*}$ is a weak solution on $\Omega$ in the sense of the variational formulation (9) considered with $\mu^{*}$, and by the uniqueness of the weak solution on $\Omega,\left.u^{*}\right|_{\Omega}=u\left(\Omega, \mu^{*}\right)$. In order to conclude that the infimum of $J$ is realized, we shall prove that

$$
\inf _{\Omega \in U_{a d}\left(\Omega_{0}, \varepsilon, \hat{c}, G\right)} J(\Omega, u(\Omega, \lambda), \lambda)=\lim _{n \rightarrow+\infty} J\left(\Omega_{n}, u_{n}\left(\Omega_{n}, \lambda\right), \lambda\right)=J\left(\Omega, u\left(\Omega, \mu^{*}\right), \mu^{*}\right) .
$$

Let us start by showing that $\left(\left.\mathbb{1}_{\Omega_{n}} E u_{n}\right|_{D}\right)_{n \in \mathbb{N}^{*}}$ converges strongly to $\left.\mathbb{1}_{\Omega} E u\right|_{D}$ in $V(D)$. Firstly, we find in the same way as previously (see [13]) that

$$
\left.\left|\int_{\Gamma_{n}}\right| \operatorname{Tr} w_{n}\right|^{2} d \lambda-\int_{\Gamma}|\operatorname{Tr} w|^{2} d \mu^{*} \mid \rightarrow 0 \quad \text { for } n \rightarrow+\infty .
$$

Then, once again, by the fact that the weak convergence of $\left(\left.E u_{n}\right|_{D}\right)_{n \in \mathbb{N}^{*}}$ to $\left.E u\right|_{D}$ in $V(D)$ implies the strong convergence in $L_{2}(D)$ and by Eqs. (25), (30), we obtain

$$
\begin{aligned}
& \lim _{n \rightarrow+\infty}\left\|\left.\mathbb{1}_{\Omega_{n}} E u_{n}\right|_{D}\right\|_{V(D, \lambda)}^{2}=\lim _{n \rightarrow+\infty}\left(\omega^{2}\left\|\left.\mathbb{1}_{\Omega_{n}} E u_{n}\right|_{D}\right\|_{L_{2}(D)}^{2}-i \int_{\Gamma_{n}} \operatorname{Im} \alpha\left|\operatorname{Tr} u_{n}\right|^{2} d \lambda\right. \\
& \left.-\int_{D} \mathbb{1}_{\Omega_{n}} f \overline{\left.E u_{n}\right|_{D}} \mathrm{~d} x+\int_{\Gamma_{n}} \operatorname{Tr} h \overline{\operatorname{Tr} u_{n}} d \lambda\right)=\omega^{2}\left\|\left.\mathbb{1}_{\Omega} E u\right|_{D}\right\|_{L_{2}(D)}^{2} \\
& -i \int_{\Gamma} \operatorname{Im} \alpha|\operatorname{Tr} u|^{2} d \mu^{*}-\int_{D} \mathbb{1}_{\Omega} f \overline{\left.E u\right|_{D}} \mathrm{~d} x+\int_{\Gamma} \operatorname{Tr} h \overline{\operatorname{Tr} u} d \mu^{*}=\|u\|_{V\left(\Omega, \mu^{*}\right)}^{2} .
\end{aligned}
$$

Since we have at the same time the weak convergence and the convergence of norms, it implies the strong convergence in $V(D)$ of $\left(\left.\mathbb{1}_{\Omega_{n}} E u_{n}\right|_{D}\right)_{n \in \mathbb{N}^{*}}$ to $\left.\mathbb{1}_{\Omega} E u\right|_{D}$.

Hence, as the functional $J$, which can be considered as an equivalent norm on $V(D)$, is continuous:

$$
\lim _{n \rightarrow+\infty} J\left(\Omega_{n}, u_{n}\left(\Omega_{n}, \lambda\right), \lambda\right)=J\left(\Omega, u\left(\Omega, \mu^{*}\right), \mu^{*}\right),
$$

i.e., as $\Omega_{n}$ is a minimizing sequence of $J, J\left(\Omega, u\left(\Omega, \mu^{*}\right), \mu^{*}\right)$ is the infimum for all $\Omega \in$ $U_{a d}\left(\Omega_{0}, \varepsilon, \hat{c}, G\right)$.

By the relation $\int_{\Gamma} d \mu^{*} \geq \int_{\Gamma} d \lambda$ we directly have

$$
J\left(\Omega, u\left(\Omega, \mu^{*}\right), \mu^{*}\right) \geq J\left(\Omega, u\left(\Omega, \mu^{*}\right), \lambda\right) .
$$

If we have $\mu^{*}=\lambda$, then $J(\Omega, u(\Omega, \lambda), \lambda)$ is the minimum.

\section{Conclusion}

Started by the well-posedness result on a large class of domains with $d$-set boundaries including even fractal boundaries, we showed that the problem of finding an optimal shape for the Helmholtz problem with a dissipative boundary has at least one solution in the sense of a suitable measure $\mu^{*}$.

\section{Acknowledgments}

We would like to thank for fruitful discussions and for a strong support of our work Profs. M.R. Lancia, M. Hinz and especially A. Teplyaev for his helpful remarks and advice, crucial in the writing of the article. We also thank our anonymous referee for pointing the interest and main difficulties in the developing of our shape optimal existence theory. 


\section{A $d$-sets and trace theorems on a $d$-set}

Let us define the main notions which we use in Theorem 1.

Definition 1 (Ahlfors $d$-regular set or $d$-set [30]). Let $F$ be a closed Borel non-empty subset of $\mathbb{R}^{N}$. The set $F$ is is called a $d$-set $(0<d \leq N)$ if there exists a $d$-measure $\mu$ on $F$, i.e. a positive Borel measure with support $F(\operatorname{supp} \mu=F)$ such that there exist constants $c_{1}$, $c_{2}>0$,

$$
c_{1} r^{d} \leq \mu(\overline{B(x, r)}) \leq c_{2} r^{d}, \quad \text { for } \forall x \in F, 0<r \leq 1,
$$

where $B(x, r) \subset \mathbb{R}^{N}$ denotes the Euclidean ball centered at $x$ and of radius $r$.

As [30, Prop. 1, p 30] all $d$-measures on a fixed $d$-set $F$ are equivalent, it is also possible to define a $d$-set by the $d$-dimensional Hausdorff measure $m_{d}$ :

$$
c_{1} r^{d} \leq m_{d}(F \cap \overline{B(x, r)}) \leq c_{2} r^{d}, \quad \text { for } \forall x \in F, 0<r \leq 1
$$

which in particular implies that $F$ has Hausdorff dimension $d$ in the neighborhood of each point of $F[30$, p.33].

If the boundary $\partial \Omega$ is a $d$-set endowed with a $d$-measure $\mu$, then we denote by $L_{2}(\partial \Omega, \mu)$ the Lebesgue space defined with respect to this measure with the norm

$$
\|u\|_{L_{2}(\partial \Omega, \mu)}=\left(\int_{\partial \Omega}|u|^{2} d \mu\right)^{\frac{1}{2}} .
$$

In particular, $N$-sets $(d$-set with $d=N)$ satisfy

$$
\exists c>0 \quad \forall x \in \bar{\Omega}, \forall r \in] 0, \delta[\cap] 0,1] \quad \lambda(B(x, r) \cap \Omega) \geq C \lambda(B(x, r))=c r^{N},
$$

where $\lambda(A)$ denotes the Lebesgue measure of a set $A$ of $\mathbb{R}^{N}$. This property is also called the measure density condition [27]. Let us notice that an $N$-set $\Omega$ cannot be "thin" close to its boundary $\partial \Omega$.

The trace operator on a $d$-set is understood in the following way:

Definition 2 (Trace operator). For an arbitrary open set $\Omega$ of $\mathbb{R}^{N}$, the trace operator $\operatorname{Tr}$ is defined [30] for $u \in L_{l o c}^{1}(\Omega)$ by

$$
\operatorname{Tr} u(x)=\lim _{r \rightarrow 0} \frac{1}{\lambda(\Omega \cap B(x, r))} \int_{\Omega \cap B(x, r)} u(y) \mathrm{d} y,
$$

where $\lambda$ denotes the Lebesgue measure. The trace operator $\operatorname{Tr}$ is considered for all $x \in \bar{\Omega}$ for which the limit exists.

Hence, the following Theorem (see Ref. [7] Section 2) generalizes the classical results [34, 35] for domains with the Lipschitz boundaries $\partial \Omega$ :

Theorem 3. Let $\Omega$ be an admissible domain in $\mathbb{R}^{N}$ in the sense of Ref. [Y], i.e. $\Omega$ is an $N$-set, such that its boundary $\partial \Omega$ is a compact d-set with a d-measure $\mu, N-2<d<N$, and the norms $\|f\|_{H^{1}(\Omega)}$ and $\|f\|_{C_{2}^{1}(\Omega)}=\|f\|_{L_{2}(\Omega)}+\left\|f_{1, \Omega}^{\sharp}\right\|_{L_{2}(\Omega)}$ with

$$
f_{1, \Omega}^{\sharp}(x)=\sup _{r>0} r^{-1} \inf _{c \in \mathbb{R}} \frac{1}{\lambda(B(x, r))} \int_{B(x, r) \cap \Omega}|f(y)-c| \mathrm{d} y,
$$

where $\lambda$ is the $N$-dimensional Lebesgue measure, are equivalent on $H^{1}(\Omega)$. Then,

1. $H^{1}(\Omega)$ is compactly embedded in $L_{2}^{\text {loc }}(\Omega)$ or in $L_{2}(\Omega)$ if $\Omega$ is bounded;

2. $\operatorname{Tr}_{\Omega}: H^{1}\left(\mathbb{R}^{N}\right) \rightarrow H^{1}(\Omega)$ is a linear continuous and surjective operator with linear bounded inverse (the extension operator $E_{\Omega}: H^{1}(\Omega) \rightarrow H^{1}\left(\mathbb{R}^{N}\right)$ ); 
3. for $\beta=1-(N-d) / 2>0$ the operators $\operatorname{Tr}: H^{1}\left(\mathbb{R}^{N}\right) \rightarrow L_{2}(\partial \Omega)$, and $\operatorname{Tr} \partial \Omega: H^{1}(\Omega) \rightarrow$ $L_{2}(\partial \Omega)$ are linear compact operators with dense image $\operatorname{Im}(\operatorname{Tr})=\operatorname{Im}\left(\operatorname{Tr}_{\partial \Omega}\right)=B_{\beta}^{2,2}(\partial \Omega)$ and with linear bounded right inverse (the extension operators) $E: B_{\beta}^{2,2}(\partial \Omega) \rightarrow$ $H^{1}\left(\mathbb{R}^{N}\right)$ and $E_{\partial \Omega}: B_{\beta}^{2,2}(\partial \Omega) \rightarrow H^{1}(\Omega) ;$

4. the Green formula holds for all $u$ and $v$ from $H^{1}(\Omega)$ with $\Delta u \in L_{2}(\Omega)$ :

$$
\int_{\Omega} v \Delta u \mathrm{~d} x+\int_{\Omega} \nabla v \cdot \nabla u \mathrm{~d} x=\left\langle\frac{\partial u}{\partial \nu}, \operatorname{Tr} v\right\rangle_{\left(\left(B_{\beta}^{2,2}(\partial \Omega)\right)^{\prime}, B_{\beta}^{2,2}(\partial \Omega)\right)},
$$

where the dual Besov space $\left(B_{\beta}^{2,2}(\partial \Omega)\right)^{\prime}=B_{-\beta}^{2,2}(\partial \Omega)$ is introduced in Ref. [31].

5. the usual integration by parts holds for all $u$ and $v$ from $H^{1}(\Omega)$ in the following weak sense

$$
\left\langle u \nu_{i}, v\right\rangle_{\left.\left(B_{\beta}^{2,2}(\partial \Omega)\right)^{\prime}, B_{\beta}^{2,2}(\partial \Omega)\right)}:=\int_{\Omega} \frac{\partial u}{\partial x_{i}} v \mathrm{~d} x+\int_{\Omega} u \frac{\partial v}{\partial x_{i}} \mathrm{~d} x \quad i=1, \ldots, N,
$$

where by $u \nu_{i}$ is denoted the linear continuous functional on $B_{\beta}^{2,2}(\partial \Omega)$.

6. $\|u\|_{H^{1}(\Omega)}$ is equivalent to $\|u\|_{\operatorname{Tr}}=\left(\int_{\Omega}|\nabla u|^{2} \mathrm{~d} x+\int_{\partial \Omega}|\operatorname{Tr} u|^{2} d \mu\right)^{\frac{1}{2}}$.

Theorem 3 is a particular case of the results proven in Ref. [7]. We also notice that in the framework of the Sobolev space $H^{1}$ and the Besov spaces $B_{\beta}^{2,2}$ with $\beta<1$, as here, we do not need to impose Markov's local inequality on $\partial \Omega$, as it is trivially satisfied (see Ref. [32] p. 198). To prove formula (33) we follow the proof of formula (4.11) of Theorem 4.5 in [16] using the existence of a sequence of domains $\left(\Omega_{m}\right)_{m \in \mathbb{N}^{*}}$ with Lipschitz boundaries such that $\Omega_{m} \subset \Omega_{m+1}$ and $\Omega=\cup_{m=1}^{\infty} \Omega_{m}$.

\section{B Approximation of the damping parameter $\alpha$ in the Robin boundary condition by a model with dissipa- tion in the volume}

Theorem 4. Let $\Omega=]-L, L[\times]-\ell, \ell[$ be a domain with a simply connected sub-domain $\Omega_{0}$, whose boundaries are $]-L, 0[\times\{\ell\},\{-L\} \times]-\ell, \ell[]-L,, 0[\times\{-\ell\}$ and another boundary, denoted by $\Gamma$, which is the straight line starting in $(0,-\ell)$ and ending in $(0, \ell)$. In addition let $\Omega_{1}$ be the supplementary domain of $\Omega_{0}$ in $\Omega$, so that $\Gamma$ is the common boundary of $\Omega_{0}$ and $\Omega_{1}$. The length $L$ is supposed to be large enough.

Let the original problem (the frequency version of the wave damped problem (1)) be

$$
\begin{aligned}
& -\nabla \cdot\left(\eta_{0} \nabla u_{0}\right)-\omega^{2} \xi_{0} u_{0}=0 \quad \text { in } \Omega_{0}, \\
& -\nabla \cdot\left(\eta_{1} \nabla u_{1}\right)-\omega^{2} \tilde{\xi}_{1} u_{1}=0 \text { in } \Omega_{1},
\end{aligned}
$$

with

$$
\tilde{\xi}_{1}=\xi_{1}\left(1+\frac{a i}{\xi_{1} \omega}\right)
$$

together with boundary conditions on $\Gamma$

$$
u_{0}=u_{1} \text { and } \eta_{0} \nabla u_{0} \cdot n=\eta_{1} \nabla u_{1} \cdot n,
$$

and the condition on the left boundary

$$
u_{0}(-L, y)=g(y)
$$


and some other boundary conditions. Let the modified problem be

$$
-\nabla \cdot\left(\eta_{0} \nabla u_{2}\right)-\omega^{2} \xi_{0} u_{2}=0 \text { in } \Omega_{0}
$$

with boundary absorption condition on $\Gamma$

$$
\eta_{0} \nabla u_{2} \cdot n+\alpha u_{2}=0
$$

and the condition on the left boundary

$$
u_{2}(-L, y)=g(y) .
$$

Let $u_{0}, u_{1}, u_{2}$ and $g$ be decomposed into Fourier modes in the $y$ direction, denoting by $k$ the associated wave number. Then the complex parameter $\alpha$, minimizing the following expression

$$
A\left\|u_{0}-u_{2}\right\|_{L_{2}\left(\Omega_{0}\right)}^{2}+B\left\|\nabla\left(u_{0}-u_{2}\right)\right\|_{L_{2}\left(\Omega_{0}\right)}^{2}
$$

can be found from the minimization of the error function

$$
e(\alpha):=\sum_{k=\frac{n \pi}{L}, n \in \mathbb{Z}} e_{k}(\alpha),
$$

where $e_{k}$ are given by

$$
\begin{aligned}
& \begin{array}{c}
e_{k}(\alpha)=\left(A+B|k|^{2}\right) \\
\left(\frac { 1 } { 2 \lambda _ { 0 } } \left\{|\chi|^{2}\left[1-\exp \left(-2 \lambda_{0} L\right)\right]\right.\right. \\
\left.\left.\quad+|\gamma|^{2}\left[\exp \left(2 \lambda_{0} L\right)-1\right]\right\}+2 L \operatorname{Re}(\chi \bar{\gamma})\right) \\
\quad+B \frac{\lambda_{0}}{2}\left\{|\chi|^{2}\left[1-\exp \left(-2 \lambda_{0} L\right)\right]+|\gamma|^{2}\left[\exp \left(2 \lambda_{0} L\right)-1\right]\right\}-2 B \lambda_{0}^{2} L \operatorname{Re}(\chi \bar{\gamma}) \\
\text { if } k^{2} \geq \frac{\xi_{0}}{\eta_{0}} \omega^{2} \text { or } \\
e_{k}(\alpha)=\left(A+B|k|^{2}\right)\left(L\left(|\chi|^{2}+|\gamma|^{2}\right)+\frac{i}{\lambda_{0}} \operatorname{Im}\left\{\chi \bar{\gamma}\left[1-\exp \left(-2 \lambda_{0} L\right)\right]\right\}\right) \\
+B L\left|\lambda_{0}\right|^{2}\left(|\chi|^{2}+|\gamma|^{2}\right)+i B \lambda_{0} \operatorname{Im}\left\{\chi \bar{\gamma}\left[1-\exp \left(-2 \lambda_{0} L\right)\right]\right\}
\end{array}
\end{aligned}
$$

if $k^{2}<\frac{\xi_{0}}{\eta_{0}} \omega^{2}$, in which

$$
\begin{aligned}
& f(x)=\left(\lambda_{0} \eta_{0}-x\right) \exp \left(-\lambda_{0} L\right)+\left(\lambda_{0} \eta_{0}+x\right) \exp \left(\lambda_{0} L\right), \\
& \chi(k, \alpha)=g_{k}\left(\frac{\lambda_{0} \eta_{0}-\lambda_{1} \eta_{1}}{f\left(\lambda_{1} \eta_{1}\right)}-\frac{\lambda_{0} \eta_{0}-\alpha}{f(\alpha)}\right), \\
& \gamma(k, \alpha)=g_{k}\left(\frac{\lambda_{0} \eta_{0}+\lambda_{1} \eta_{1}}{f\left(\lambda_{1} \eta_{1}\right)}-\frac{\lambda_{0} \eta_{0}+\alpha}{f(\alpha)}\right)
\end{aligned}
$$

where

$$
\left\{\begin{array}{lll}
\lambda_{0}=\sqrt{k^{2}-\frac{\xi_{0}}{\eta_{0}} \omega^{2}} & \text { if } & k^{2} \geq \frac{\xi_{0}}{\eta_{0}} \omega^{2} \\
\lambda_{0}=i \sqrt{\frac{\xi_{0}}{\eta_{0}} \omega^{2}-k^{2}} & \text { if } & k^{2} \leq \frac{\xi_{0}}{\eta_{0}} \omega^{2} .
\end{array}\right.
$$

Proof. First of all,

$$
e(\alpha):=A\left\|u_{0}-u_{2}\right\|_{L_{2}\left(\Omega_{0}\right)}^{2}+B\left\|\nabla\left(u_{0}-u_{2}\right)\right\|_{L_{2}\left(\Omega_{0}\right)}^{2}
$$

can be decomposed as a sum of $e_{k}(\alpha)$

$$
e(\alpha):=\sum_{k=\frac{n \pi}{L}, n \in \mathbb{Z}} e_{k}(\alpha),
$$


with

$$
e_{k}(\alpha)=A\left\|u_{0, k}-u_{2, k}\right\|_{L_{2}(]-L, 0[)}^{2}+B\left\|\nabla\left(u_{0, k}-u_{2, k}\right)\right\|_{L_{2}(]-L, 0[)}^{2},
$$

where we have decomposed decomposed $u_{0}, u_{1}$ and $u_{2}$ into modes in the $y$ direction, denoting by $k$ the associated wave number.

The mode $u_{0, k}$ solves

$$
\partial_{x x} u_{0, k}-\left(k^{2}-\frac{\xi_{0}}{\eta_{0}} \omega^{2}\right) u_{0, k}=0
$$

and thus

$$
u_{0, k}(x)=A_{0} \exp \left(\lambda_{0} x\right)+B_{0} \exp \left(-\lambda_{0} x\right),
$$

where $\lambda_{0}$ is given in Eq. (41).

The mode $u_{1, k}$ solves

$$
\partial_{x x} u_{1, k}-\left(k^{2}-\frac{\tilde{\xi}_{1}}{\eta_{1}} \omega^{2}\right) u_{1, k}=0,
$$

and thus

$$
u_{1, k}(x)=A_{1} \exp \left(\lambda_{1} x\right)+B_{1} \exp \left(-\lambda_{1} x\right),
$$

where

$$
\lambda_{1}^{2}=k^{2}-\left(1+\frac{a i}{\xi_{1} \omega}\right) \frac{\xi_{1}}{\eta_{1}} \omega^{2}
$$

so that

$$
\begin{array}{r}
\lambda_{1}=\frac{1}{\sqrt{2}} \sqrt{k^{2}-\frac{\xi_{1}}{\eta_{1}} \omega^{2}+\sqrt{\left(k^{2}-\frac{\xi_{1}}{\eta_{1}} \omega^{2}\right)^{2}+\left(\frac{a \omega}{\eta_{1}}\right)^{2}}} \\
-\frac{i}{\sqrt{2}} \sqrt{\frac{\xi_{1}}{\eta_{1}} \omega^{2}-k^{2}+\sqrt{\left(k^{2}-\frac{\xi_{1}}{\eta_{1}} \omega^{2}\right)^{2}+\left(\frac{a \omega}{\eta_{1}}\right)^{2}}} .
\end{array}
$$

For large $L$, since $\operatorname{Re}\left(\lambda_{1}\right)>0$, the value of $A_{1}$ tend to 0 , so that we may neglect the first contribution in the right-hand side of Eq. (43). Consequently we consider the expression

$$
u_{1, k}(x)=B_{1} \exp \left(-\lambda_{1} x\right) .
$$

Continuity conditions (36) and expressions (42) and (44) imply the following relations

$$
A_{0}+B_{0}=B_{1}, \eta_{0} \lambda_{0}\left(A_{0}-B_{0}\right)=-\eta_{1} \lambda_{1} B_{1},
$$

from which we infer that

$$
B_{0}=\frac{\lambda_{0} \eta_{0}+\lambda_{1} \eta_{1}}{\lambda_{0} \eta_{0}-\lambda_{1} \eta_{1}} A_{0}
$$

and thus

$$
u_{0, k}(x)=A_{0}\left[\exp \left(\lambda_{0} x\right)+\frac{\lambda_{0} \eta_{0}+\lambda_{1} \eta_{1}}{\lambda_{0} \eta_{0}-\lambda_{1} \eta_{1}} \exp \left(-\lambda_{0} x\right)\right] .
$$

The decomposition of the boundary condition (37) into Fourier modes implies that $u_{0, k}(-L)=$ $g_{k}$, which gives the final expression

$$
u_{0, k}(x)=g_{k} \frac{\left[\left(\lambda_{0} \eta_{0}-\lambda_{1} \eta_{1}\right) \exp \left(\lambda_{0} x\right)+\left(\lambda_{0} \eta_{0}+\lambda_{1} \eta_{1}\right) \exp \left(-\lambda_{0} x\right)\right]}{\left[\left(\lambda_{0} \eta_{0}-\lambda_{1} \eta_{1}\right) \exp \left(-\lambda_{0} L\right)+\left(\lambda_{0} \eta_{0}+\lambda_{1} \eta_{1}\right) \exp \left(\lambda_{0} L\right)\right]} .
$$

Let us now turn to the expression of $u_{2, k}$. Since equation (38) is the same as that verified by $u_{0, k}$, both solutions have the same general form:

$$
u_{2, k}(x)=A_{2} \exp \left(\lambda_{0} x\right)+B_{2} \exp \left(-\lambda_{0} x\right) .
$$


The Robin boundary condition (39) on $\Gamma$ implies that

$$
\eta_{0} \lambda_{0}\left(A_{2}-B_{2}\right)+\alpha\left(A_{2}+B_{2}\right)=0
$$

which means that

$$
u_{2, k}(x)=A_{2}\left[\exp \left(\lambda_{0} x\right)+\frac{\lambda_{0} \eta_{0}+\alpha}{\lambda_{0} \eta_{0}-\alpha} \exp \left(-\lambda_{0} x\right)\right] .
$$

Application of the boundary condition (40) implies the final expression

$$
u_{2, k}(x)=g_{k} \frac{\left[\left(\lambda_{0} \eta_{0}-\alpha\right) \exp \left(\lambda_{0} x\right)+\left(\lambda_{0} \eta_{0}+\alpha\right) \exp \left(-\lambda_{0} x\right)\right]}{\left[\left(\lambda_{0} \eta_{0}-\alpha\right) \exp \left(-\lambda_{0} L\right)+\left(\lambda_{0} \eta_{0}+\alpha\right) \exp \left(\lambda_{0} L\right)\right]} .
$$

Using (45) and (46), we have that

$$
\left(u_{0, k}-u_{2, k}\right)(x)=\chi(k, \alpha) \exp \left(\lambda_{0} x\right)+\gamma(k, \alpha) \exp \left(-\lambda_{0} x\right),
$$

where the coefficients $\chi$ and $\gamma$ are computed from (45) and (46). In order to compute the $L_{2}$ norm of this expression, we must first compute the square of its modulus (by $\bar{\gamma}$ is denoted the complex conjugate of $\gamma$ ):

$$
\left|u_{0, k}-u_{2, k}\right|^{2}(x)=|\chi|^{2}\left|\exp \left(\lambda_{0} x\right)\right|^{2}+|\gamma|^{2}\left|\exp \left(-\lambda_{0} x\right)\right|^{2}+2 \operatorname{Re}\left(\chi \bar{\gamma} \exp \left(\lambda_{0} x\right) \overline{\exp \left(-\lambda_{0} x\right)}\right) .
$$

Note that, according to the values of $k$, the expression above may be simplified into

$$
\left|u_{0, k}-u_{2, k}\right|^{2}(x)=|\chi|^{2} \exp \left(2 \lambda_{0} x\right)+|\gamma|^{2} \exp \left(-2 \lambda_{0} x\right)+2 \operatorname{Re}(\chi \bar{\gamma})
$$

if $k^{2} \geq \frac{\xi_{0}}{\eta_{0}} \omega^{2}$, or

$$
\left|u_{0, k}-u_{2, k}\right|^{2}(x)=|\chi|^{2}+|\gamma|^{2}+2 \operatorname{Re}\left(\chi \bar{\eta} \exp \left(2 \lambda_{0} x\right)\right)
$$

if $k^{2}<\frac{\xi_{0}}{\eta_{0}} \omega^{2}$. Thus, we have for $k^{2} \geq \frac{\xi_{0}}{\eta_{0}} \omega^{2}$

$$
\begin{gathered}
\int_{-L}^{0}\left|u_{0, k}-u_{2, k}\right|^{2}(x) \mathrm{d} x=\frac{1}{2 \lambda_{0}}\left\{|\chi|^{2}\left[1-\exp \left(-2 \lambda_{0} L\right)\right]+|\gamma|^{2}\left[\exp \left(2 \lambda_{0} L\right)-1\right]\right\} \\
+2 L \operatorname{Re}(\chi \bar{\gamma})
\end{gathered}
$$

or, for $k^{2}<\frac{\xi_{0}}{\eta_{0}} \omega^{2}$

$$
\int_{-L}^{0}\left|u_{0, k}-u_{2, k}\right|^{2}(x) \mathrm{d} x=L\left(|\chi|^{2}+|\gamma|^{2}\right)+\frac{i}{\lambda_{0}} \operatorname{Im}\left\{\chi \bar{\gamma}\left[1-\exp \left(-2 \lambda_{0} L\right)\right]\right\} \text {. }
$$

Now, we also have to compute the $L_{2}$ norm of the gradient of $\left(u_{0, k}-u_{2, k}\right)$. Noting that

$$
\nabla\left(u_{0, k}-u_{2, k}\right)=\left(\begin{array}{c}
\partial_{x}\left(u_{0, k}-u_{2, k}\right) \\
i k\left(u_{0, k}-u_{2, k}\right)
\end{array}\right)
$$

it holds that

$$
\left|\nabla\left(u_{0, k}-u_{2, k}\right)\right|^{2}=|k|^{2}\left|u_{0, k}-u_{2, k}\right|^{2}+\left|\partial_{x}\left(u_{0, k}-u_{2, k}\right)\right|^{2} .
$$

With expression (47), it follows that

$$
\left|\partial_{x}\left(u_{0, k}-u_{2, k}\right)\right|^{2}=\left|\lambda_{0}\right|^{2}\left[|\chi|^{2} \exp \left(2 \lambda_{0} x\right)+|\gamma|^{2} \exp \left(-2 \lambda_{0} x\right)-2 \operatorname{Re}(\chi \bar{\gamma})\right],
$$

if $k^{2} \geq \frac{\xi_{0}}{\eta_{0}} \omega^{2}$, or

$$
\left|\partial_{x}\left(u_{0, k}-u_{2, k}\right)\right|^{2}=\left|\lambda_{0}\right|^{2}\left[|\chi|^{2}+|\gamma|^{2}-2 \operatorname{Re}\left(\chi \bar{\gamma} \exp \left(2 \lambda_{0} x\right)\right)\right],
$$


if $k^{2}<\frac{\xi_{0}}{\eta_{0}} \omega^{2}$, and thus

$$
\begin{array}{r}
\int_{-L}^{0}\left|\partial_{x}\left(u_{0, k}-u_{2, k}\right)\right|^{2}(x) \mathrm{d} x=\frac{\lambda_{0}}{2}\left\{|\chi|^{2}\left[1-\exp \left(-2 \lambda_{0} L\right)\right]+|\gamma|^{2}\left[\exp \left(2 \lambda_{0} L\right)-1\right]\right\} \\
-2 \lambda_{0}^{2} L \operatorname{Re}(\chi \bar{\gamma})
\end{array}
$$

if $k^{2} \geq \frac{\xi_{0}}{\eta_{0}} \omega^{2}$, or, if $k^{2}<\frac{\xi_{0}}{\eta_{0}} \omega^{2}$,

$$
\int_{-L}^{0}\left|\partial_{x}\left(u_{0, k}-u_{2, k}\right)\right|^{2}(x) \mathrm{d} x=L\left|\lambda_{0}\right|^{2}\left(|\chi|^{2}+|\gamma|^{2}\right)+i \lambda_{0} \operatorname{Im}\left\{\chi \bar{\gamma}\left[1-\exp \left(-2 \lambda_{0} L\right)\right]\right\} .
$$

Therefore, we can find $\alpha$ as the solution of the mentioned minimization problem.

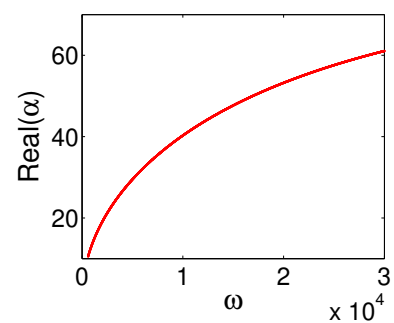

(a) $\operatorname{Re}(\alpha)$

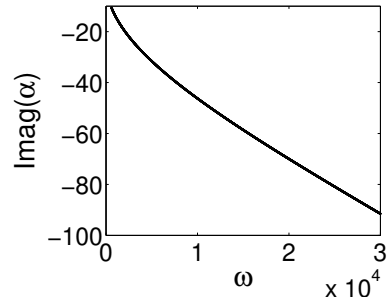

(b) $\operatorname{Im}(\alpha)$

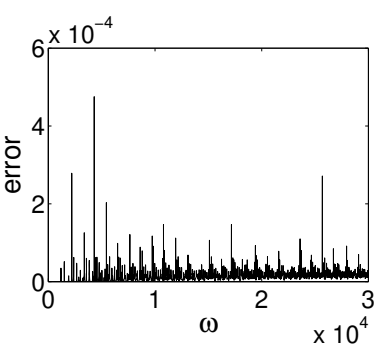

(c) error

Figure 2: The real (top left) and imaginary (top right) parts of $\alpha$ and the sum of the errors $e_{\Delta x}$ (in the bottom) as function of frequencies $\omega \in[600,30000]$ calculated for the ISOREL porous material.

Since the minimization will be done numerically and since the sequence $(z,-z, z-$ $z, \cdots)=z(\exp (i(j \Delta x) / \Delta x))$ is the highest frequency mode that can be reached on a grid of size $\Delta x$, then, in practice, the sum may be truncated to

$$
e_{\Delta x}(\alpha):=\sum_{k=\frac{n \pi}{L}, n \in \mathbb{Z},-\frac{L}{\Delta x} \leq n \leq \frac{L}{\Delta x}} e_{k}(\alpha) .
$$

For the equations (34)-(35), we use the same coefficients as for problem (1) and take the values corresponding to a porous medium, called ISOREL, using in building insulation. More precisely we assume: $\phi=0.7, \gamma_{p}=1.4, \sigma=142300 \mathrm{~N} . \mathrm{m}^{-4} . \mathrm{s}, \rho_{0}=1.2 \mathrm{~kg} / \mathrm{m}^{3}, \alpha_{h}=1.15$, $c_{0}=340 \mathrm{~m} . \mathrm{s}^{-1}$. We could find the value of $\alpha$ presented in Fig. 2.

Remark 2. Fig. 2 allows us to compare the difference between two considered time-dependent models for the damping in the volume and for the damping on the boundary. We see that $\operatorname{Re}(\alpha)$ is not a constant in general, but for $\omega \rightarrow+\infty \operatorname{Im}(\alpha)$ is a linear function of $\omega$. In this sense, the damping properties of two models are almost the same, but the reflection is more accurately considered by the damping wave equation in the volume.

\section{References}

[1] K. ABe, T. Fujiu, And K. Koro, A BE-based shape optimization method enhanced by topological derivative for sound scattering problems, Engineering Analysis with Boundary Elements, 34 (2010), pp. 1082-1091, doi:10.1016/j.enganabound.2010.06.017. 
[2] Y. Achdou and O. Pironneau, Optimization of a photocell, Optimal Control Applications and Methods, 12 (1991), pp. 221-246, doi:10.1002/oca.4660120403.

[3] S. Agmon, Lectures on elliptic boundary value problems, Van Mostrand Math. Studies, 1965.

[4] G. Allaire, Conception optimale de structures, 58 Mathématiques et Applications, Springer, 2007.

[5] L. Ambrosio, N. Fusco, And D. Pallara, Functions of bounded variation and free discontinuity problems, Oxford Mathematical Monographs, 2000.

[6] H. Antil, S. Hardesty, and M. Heinkenschloss, Shape Optimization of Shell Structure Acoustics, SIAM Journal on Control and Optimization, 55 (2017), pp. 13471376, doi:10.1137/16M1070633.

[7] K. Arfi and A. Rozanova Pierrat, Dirichlet-to-Neumann or Poincaré-Steklov operator on fractals described by $d$-sets, Discrete and Continuous Dynamical Systems - S, 12 (2019), pp. 1-26.

[8] M. Asch And G. Lebeau, The Spectrum of the Damped Wave Operator for a Bounded Domain in $\mathbb{R}^{2}$, Experimental Mathematics, 12 (2003), pp. 227-241, doi:10.1080/10586458.2003.10504494.

[9] C. BARdos AND J. RAUCH, Variational algorithms for the Helmholtz equation using time evolution and artificial boundaries, Asymptotic Analysis, 9 (1994), pp. 101-117.

[10] D. Bucur And A. Giacomini, Shape optimization problems with Robin conditions on the free boundary, Annales de l'Institut Henri Poincaré C, Analyse non linéaire, 33 (2016), pp. 1539-1568, doi:10.1016/j.anihpc.2015.07.001.

[11] Y. CaO And D. Stanescu, Shape optimization for noise radiation problems, Computers \& Mathematics with Applications, 44 (2002), pp. 1527-1537, doi:10.1016/S08981221(02)00276-6.

[12] R. Capitanelli, Asymptotics for mixed Dirichlet-Robin problems in irregular domains, Journal of Mathematical Analysis and Applications, 362 (2010), pp. 450-459, doi:10.1016/j.jmaa.2009.09.042.

[13] R. Capitanelli, Robin boundary condition on scale irregular fractals, Communications on Pure and Applied Analysis, 9 (2010), pp. 1221-1234, doi:10.3934/cpaa.2010.9.1221.

[14] D. Chenais, On the existence of a solution in a domain identification problem, Journal of Mathematical Analysis and Applications, 52 (1975), pp. 189-219, doi:10.1016/0022247X(75)90091-8.

[15] S. Cox AND E. ZuAzuA, The rate at which energy decays in a damped string, Communications in Partial Differential Equations, 19 (1994), pp. 213-243, doi:10.1080/03605309408821015.

[16] S. Creo, M. R. Lancia, P. Vernole, M. Hinz, and A. Teplyaev, Magnetostatic problems in fractal domains, (2018), arXiv:1805.08262v2.

[17] J. DARDÉ, Méthodes de quasi-réversibilité et de lignes de niveau appliquées aux problèmes inverses elliptiques, $\mathrm{PhD}$ thesis, 2010.

[18] R. Dudley, Real Analysis and Probability, The Wadsworth \& Brooks Cole Mathematics Series, Pacific Grove, California, 1989.

[19] D. Duhamel, Calcul de murs antibruit et control actif du son, $\mathrm{PhD}$ thesis, Mémoire dhabilitation, 1998. 
[20] D. Duhamel, Shape optimization of noise barriers using genetic algorithms, Journal of Sound and Vibration, 297 (2006), pp. 432-443, doi:10.1016/j.jsv.2006.04.004.

[21] L. C. Evans, Partial Differential Equations, American Math Society, 2010.

[22] K. J. Falconer, The Geometry of Fractal Sets, Cambridge Tracts in Maths, 1985.

[23] B. Farhadinia, An Optimal Shape Design Problem for Fan Noise Reduction, JSEA, 03 (2010), pp. 610-613, doi:10.4236/jsea.2010.36071.

[24] S. FÉlix, M. Asch, M. Filoche, AND B. SAPoval, Localization and increased damping in irregular acoustic cavities, Journal of Sound and Vibration, 299 (2007), pp. 965976, doi:10.1016/j.jsv.2006.07.036.

[25] M. J. Gander, L. Halpern, and F. Magoulès, An optimized Schwarz method with two-sided Robin transmission conditions for the Helmholtz equation, International Journal for Numerical Methods in Fluids, 55 (2007), pp. 163-175, doi:10.1002/fld.1433.

[26] D. Guicking, On the invention of active noise control by Paul Lueg, The Journal of the Acoustical Society of America, 87 (1990), p. 2251, doi:10.1121/1.399195.

[27] P. HajŁasz, P. Koskela, and H. Tuominen, Sobolev embeddings, extensions and measure density condition, Journal of Functional Analysis, 254 (2008), pp. 1217-1234, doi:10.1016/j.jfa.2007.11.020.

[28] J.-F. Hamet And M. Berengier, Acoustical characteristics of porous pavements: a new phenomenological model, Internoise 93,Louvain, Belgique, (1993), pp. 641-646.

[29] A. Henrot And M. Pierre, Variation et optimization de formes. Une analyse géométrique, Springer, 2005.

[30] A. Jonsson and H. Wallin, Function spaces on subsets of $\mathbb{R}^{n}$, Math. Reports 2, Part 1, Harwood Acad. Publ. London, 1984.

[31] A. Jonsson And H. Wallin, The dual of Besov spaces on fractals, Studia Mathematica, 112 (1995), pp. 285-300.

[32] A. Jonsson And H. WAllin, Boundary value problems and brownian motion on fractals, Chaos, Solitons \& Fractals, 8 (1997), pp. 191-205, doi:10.1016/S09600779(96)00048-3.

[33] M. R. Lancia, A Transmission Problem with a Fractal Interface, Zeitschrift für Analysis und ihre Anwendungen, 21 (2002), pp. 113-133, doi:10.4171/ZAA/1067.

[34] J. Lions and E. Magenes, Non-Homogeneous Boundary Value Problems and Applications, vol. 1, Berlin: Springer-Verlag, 1972.

[35] J. Marschall, The trace of Sobolev-Slobodeckij spaces on Lipschitz domains, Manuscripta Math, 58 (1987), pp. 47-65, doi:10.1007/BF01169082.

[36] V. MaZ’JA, Sobolev Spaces, Springer Ser. Sov. Math., Springer-Verlag, Berlin,, 1985.

[37] B. Mohammadi and O. Pironneau, Applied shape optimization for fluids, Oxford University Press, 2010.

[38] A. MÜNCH, Optimal Internal Dissipation of a Damped Wave Equation Using a Topological Approach, International Journal of Applied Mathematics and Computer Science, 19 (2009), doi:10.2478/v10006-009-0002-x.

[39] A. MÜnch, P. Pedregal, and F. Periago, Optimal design of the damping set for the stabilization of the wave equation, Journal of Differential Equations, 231 (2006), pp. 331-358, doi:10.1016/j.jde.2006.06.009. 
[40] A. Rozanova Pierrat, Generalization of Rellich-Kondrachov theorem and trace compacteness in the framework of irregular and fractal boundaries, Preprint hal-02489325, (2020).

[41] H. Wallin, The trace to the boundary of Sobolev spaces on a snowflake, Manuscripta Math, 73 (1991), pp. 117-125, doi:10.1007/BF02567633. 